\title{
A membrane paradigm at large $D$
}

\author{
Sayantani Bhattacharyya, ${ }^{a}$ Anandita De, ${ }^{b}$ Shiraz Minwalla, ${ }^{c}$ Ravi Mohan ${ }^{c}$ \\ and Arunabha Saha ${ }^{c}$ \\ ${ }^{a}$ Department of Physics, Indian Institute of Technology Kanpur, \\ Kanpur 208016, India \\ ${ }^{b}$ Department of Physics, Indian Institute of Science Education and Research, \\ Dr. Homi Bhabha Road, Pune 411008, India \\ ${ }^{c}$ Department of Theoretical Physics, Tata Institute of Fundamental Research, \\ Homi Bhabha Road, Mumbai 400005, India \\ E-mail: sayanta@iitk.ac.in, ananditade@students.iiserpune.ac.in, \\ minwalla@theory.tifr.res.in, ravimohan1991@gmail.com, \\ arunabha@theory.tifr.res.in
}

ABStract: We study $\mathrm{SO}(d+1)$ invariant solutions of the classical vacuum Einstein equations in $p+d+3$ dimensions. In the limit $d \rightarrow \infty$ with $p$ held fixed we construct a class of solutions labelled by the shape of a membrane (the event horizon), together with a 'velocity' field that lives on this membrane. We demonstrate that our metrics can be corrected to nonsingular solutions at first sub-leading order in $\frac{1}{d}$ if and only if the membrane shape and 'velocity' field obey equations of motion which we determine. These equations define a well posed initial value problem for the membrane shape and this 'velocity' and so completely determine the dynamics of the black hole. They may be viewed as governing the non-linear dynamics of the light quasi normal modes of Emparan, Suzuki and Tanabe.

Keywords: Black Holes, Classical Theories of Gravity

ARXiv EPrint: 1504.06613 


\section{Contents}

1 Introduction $\quad 2$

2 Membrane like solutions in the large $d$ limit $\quad 6$

2.1 Dimensional reduction of $\mathrm{SO}(d+1)$ invariant metrics 6

2.2 A large $d$ limit 7

2.3 Flat solutions of the leading large $d$ equations 8

2.4 The black brane 8

2.5 Sewing black branes into a membrane 9

2.6 Equivalent starting points for perturbation theory and a 'gauge choice' 10

3 Correction at first order in $\frac{1}{d} \quad 12$

$\begin{array}{lll}3.1 & \text { Interpretation of Taylor coefficients and constraints } & 12\end{array}$

$\begin{array}{lll}3.2 & \text { Data } & 13\end{array}$

$\begin{array}{lll}3.3 & \text { Structure of the first correction to the metric } & 13\end{array}$

$\begin{array}{lll}3.4 \text { Results } & 15\end{array}$

4 Stationary black hole solutions $\quad \mathbf{1 6}$

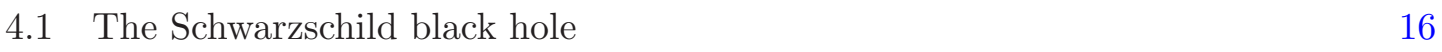

$\begin{array}{ll}4.2 & \text { The Myers-Perry black hole } \\ \end{array}$

5 Linearization of membrane equations about the Schwarzschild solution 19

$\begin{array}{ll}5.1 \text { The scalar equation } & 20\end{array}$

$\begin{array}{lll}5.2 & \text { The vector equation } & 23\end{array}$

A Technical details $\quad \mathbf{2 5}$

A.1 Simplification of the dimensionally reduced equations 25

A.2 Details of black branes 25

A.2.1 Standard coordinates for the black brane 25

A.2.2 Uniform scaling of black branes 26

$\begin{array}{lll}\text { A.3 Location of the event horizon } & 27\end{array}$

$\begin{array}{lll}\text { A.4 Equivalence in data off the membrane } & 27\end{array}$

$\begin{array}{lll}\text { A.5 Details concerning Myers-Perry black holes } 28 & 28\end{array}$

$\begin{array}{ll}\text { B Details of the perturbative procedure } & 31\end{array}$

B.1 Scalar sector $\quad 32$

B.2 Vector sector 34

B.3 Tensor sector $\quad 35$ 


\section{Introduction}

The rich classical dynamics of uncharged black holes is governed by the vacuum Einstein equation

$$
R_{\mu \nu}=0 .
$$

This innocuous looking equation captures very complicated processes; for example the collision of two black holes and their subsequent merger accompanied by gravitational radiation. We owe much of our current understanding of these processes to numerics. However the numerics involved are very challenging; their complexity makes it impractical to densely fill the space of initial conditions with numerical solutions. It would thus be useful to have analytic techniques to analyse these solutions. However the phenomena described are of such complexity that it seems unlikely that exact analytic solutions will ever be obtained. Moreover perturbation theory appears to be ruled out by the fact that Einstein's equations lack a parameter.

In this paper we follow the lead of Emparan, Suzuki and Tanabe (EST) to introduce a parameter into Einstein's equations. We do this by studying these equations in $D$ dimensions. The equations of black hole dynamics simplify in this limit and admit a systematic expansion in $\frac{1}{D}$. Our general strategy is similar to that of t'Hooft [1] who introduced a parameter into the study of Yang Mills theory by replacing the $\mathrm{SU}(3)$ gauge group by $\mathrm{SU}(N)$, and that of Witten who introduced a parameter into the study of atomic and molecular physics by analysing the quantum mechanics of the $\frac{1}{r}$ potential in large dimensions [2].

EST and collaborators have recently pointed out [3-10] that several features of black hole dynamics simplify in the limit of a large number of spacetime dimensions $D$. A Schwarzschild black hole with Schwarzschild radius $r_{0}$ in $D$ spacetime dimensions is described by the metric

$$
d s^{2}=-\left(1-\left(\frac{r_{0}}{r}\right)^{D-3}\right) d t^{2}+\frac{d r^{2}}{\left(1-\left(\frac{r_{0}}{r}\right)^{D-3}\right)}+r^{2} d \Omega_{D-2}^{2} .
$$

If $r$ is held fixed at a value greater than $r_{0}$ as $D \rightarrow \infty$ then $\left(\frac{r_{0}}{r}\right)^{D-3} \rightarrow 0$ and the metric (1.2) reduces to flat space. On the other hand if we set $r=r_{0}\left(1+\frac{R}{D-3}\right)$ and hold $R$ fixed as $D$ is taken to infinity then $\left(\frac{r_{0}}{r}\right)^{D-3} \rightarrow e^{-R}$. It follows that the gravitational tail of a black hole in $D$ spacetime dimensions extends only over a distance $\frac{r_{0}}{D-3}$ away from its event horizon [3], a distance we will refer to as the 'thickness of the membrane'.

EST have computed spectrum of quasinormal modes of the black hole (1.2) in an expansion in $\frac{1}{D}[6,8]$. They find that almost all of the infinite number of quasinormal modes at every angular momentum have frequencies of order the inverse of the membrane thickness, i.e. of order $\frac{D}{r_{0}}$. However at each angular momentum a small number of modes (two scalars and one vector at each value of the angular momentum) are much lighter than the generic mode; the frequencies of the light modes are of order $\frac{1}{r_{0}}$. Moreover the light quasinormal modes are supported entirely inside the membrane region. ${ }^{1}$

\footnotetext{
${ }^{1}$ In contrast the heavy quasinonrmal modes are non-trivial outside the membrane region where they reduce to purely outgoing modes.
} 
The separation in scale between the heavy and light quasinormal modes suggests that the full nonlinear dynamics of a black hole over time scales much larger than $\frac{r_{0}}{D}$ is governed by an effective non-linear theory of the light quasinormal modes. We expect the degrees of freedom of this theory to reside within the membrane region, and so effectively on a codimension one surface in spacetime at length scales large compared to $\frac{r_{0}}{D}$.

In this paper we will verify the expectation of the previous paragraph by explicit analysis of Einstein's equations. We identify the auxiliary dynamical system that lives on the membrane and determine its dynamical equations of motion. Our work has several similarities to the Fluid-Gravity correspondence ([11] see $[12,13]$ for reviews), however there are also significant differences. The construction of this paper applies to gravity even in flat space. The effective 'hydrodynamical' theory obtained in this paper lives on a fluctuating surface rather than the fixed field theory background of fluid gravity. Finally the constructions presented in this paper are [14] justified not by an expansion in gradients in units of the horizon radius, but rather by an expansion in $\frac{1}{D}$. Our membranes also has several conceptual similarities to the blackfolds (see e.g. [14-17]), but differ from the later in decoupling from radiation.

A solution of Einstein's equations in $D$ dimensions is given by a metric tensor with $D(D+1) / 2$ components; each of these components is a function of $D$ spacetime variables. It is clear that the generic solution of this sort has no analogue at any fixed finite value of $D$. For example a metric in four dimensions has many fewer legs and and many fewer directions in which to wiggle than a metric in a million dimensions. In order to take a sensible large $D$ limit we write $D=p+d+3$ and divide up the $D$ spacetime dimensions into two groups. The first group contains $p+2$ dimensions including time and the second contains the remaining $d+1$ dimensions. We restrict our attention only to solutions that preserve the $\mathrm{SO}(d+1)$ rotational symmetry in $d+1$ dimensions. We then take the limit $d \rightarrow \infty$ with $p$ held fixed. ${ }^{2}$

In the rest of this paper we use the following notation. Let $w_{a}$ represent the first set of $p+2$ coordinates. Let $z_{M}$ denote the last set of $d+1$ coordinates. The metric of flat $D$ dimensional spacetime is

$$
\begin{aligned}
d s^{2} & =d w_{a} d w^{a}+d z_{M} d z^{M}=d w_{a} d w^{a}+d S^{2}+S^{2} d \Omega_{d}^{2} \\
S^{2} & =z_{M} z^{M}
\end{aligned}
$$

Let $x^{\mu}=\left(S, w^{a}\right)$ so that the index $\mu$ runs over $p+3$ variables. The most general metric that preserves the $\mathrm{SO}(d+1)$ isometry takes the form

$$
d s_{\text {full }}^{2}=g_{\mu \nu} d x^{\mu} d x^{\nu}+e^{\phi} d \Omega_{d}^{2}
$$

where $g_{\mu \nu}$ and $\phi$ respectively are a metric and a scalar field in the $p+3$ dimensional space spanned by $x^{\mu}$; in particular they are functions only of $x^{\mu}$ and not of the coordinates of the $d$ sphere.

Below we derive the equations of motion for $g_{\mu \nu}$ and $\phi$, and explain that these equations admit an interesting large $d$ limit. We then explicitly construct a large family of solutions

\footnotetext{
${ }^{2}$ It follows that the solutions we study have analogues in all dimensions greater than or equal to $p+3$.
} 
of these equations. The solutions we construct are in one to one correspondence with the solutions of an auxiliary problem of membrane dynamics in flat space that we now describe.

Our auxiliary dynamical system lives on a codimension one membrane that resides in the flat space (1.3). This membrane is free to fluctuate, subject to the requirement that it preserves $\mathrm{SO}(d+1)$ invariance. The position of the membrane can always be characterized by the zeroes of a function $B\left(x^{\mu}\right)$ and $\mathrm{SO}(d+1)$ invariance forces $B\left(x^{\mu}\right)$ to be a function only of $w^{a}$ and $S$ (and not of the angular coordinates on the $d$ sphere). We could, for example, choose the function $B$ to take the form $B=S-g\left(w^{a}\right)$ so that the membrane surface is given simply by the equation $S=g\left(w^{a}\right)$. It follows that the data in the shape of the membrane surface is contained in a single function of $p+2$ variables.

For many purposes we can think of the membrane as propagating in the auxiliary flat space. $^{3}$

$$
d s^{2}=d w_{a} d w^{a}+d S^{2}
$$

Let $n_{\mu}$ denote the outward pointing unit normalized normal one form field of the membrane. $n_{\mu}$ is assumed always to be spacelike. $n_{\mu}$ is of course completely determined by the shape of the membrane surface; in terms of the function $B$ we have $n_{\mu}=\frac{d B}{\sqrt{d B \cdot d B}}$.

Our membrane is also equipped with a 'velocity' field $u_{\mu}$ on its world volume ${ }^{4} u_{\mu}$ is a $p+3$ dimensional one form field that is further constrained by the following unusual conditions

$$
\begin{aligned}
u^{2} & =-1 \\
u . n & =n_{S}-\frac{1}{n_{S}} \\
u_{S} & =0
\end{aligned}
$$

(here $n_{S}$ and $u_{S}$ are simply the $S$ components of the one forms $n$ and $u$ ). The first of (1.6) simply asserts that $u_{\mu}$ is a timelike one form of unit norm, as is usually true of a velocity field. The second of (1.6) asserts that the normal component of $u_{\mu}$ is not free but is determined by the other components of $u_{\mu}$ and the angle the membrane normal vector makes with a unit vector in the $S$ direction. The third of (1.6) asserts that the velocity field has no component in the special $S$ direction. The velocity one form field $u_{\mu}$ is specified by $p+3$ functions that live on the membrane. These $p+3$ equations are constrained by by the 3 equations (1.6) so that the data in the velocity is effectively $p$ functions of $p+2$ membrane coordinates.

The shape of the membrane and the velocity field described above are the variables of our auxiliary dynamical system. As we will demonstrate below, the equations of this

\footnotetext{
${ }^{3}$ However the $S$ coordinate is inequivalent to the other $w^{a}$ coordinates and so our effective dynamical system enjoys invariance only under the $p+2$ dimensional Lorentz invariance.

${ }^{4}$ More precisely our membrane has a null oneform field $O$ living on its world volume. It turns out that the overall scale of $O$ can be absorbed into a subleading shift of the membrane surface and so is irrelevant at leading order. Upto a scaling this oneform can be cast in the form $O=d S-u_{a} d w^{a}$; the fact that $O . O=0$ then implies that $u . u=-1$. This normalization leads us to refer to the collective coordinate $u_{\mu}$ as a 'velocity'. The precise physical significance of $u_{\mu}$ will be clear once we understand its contribution to the membrane stress tensor. We leave this to future work.
} 
system turn out to be

$$
\begin{aligned}
U_{\perp} . K . U_{\perp}+n_{S}\left(n_{S}^{2}-1\right) / S & =0 \\
\mathcal{P}_{a}^{b}\left(U_{\perp} \cdot \nabla u_{b}\right) & =0
\end{aligned}
$$

where

$$
U_{\perp}=U-(U . n) n, \quad U=d S+n_{S}^{2}\left(d S-u_{\mu} d x^{\mu}\right)
$$

$K_{\mu \nu}$ is the extrinsic curvature of the surface, the symbol dot (.) denotes contraction of indices (all indices are raised and lowered by the flat metric (1.3)) and $\mathcal{P}_{a}^{b}$ is the projector orthogonal to the 3 dimensional subspace spanned by the three one forms $u_{\mu}, n_{\mu}$ and $d S$ (so that $\mathcal{P}_{a}^{b}$ projects onto a $p$ dimensional subspace). (1.7) are a set of $p+1$ equations that define an initial value problem for $p+1$ variables (the shape of the surface and the constrained one form field $u_{\mu}$ ). These equations are of first order in time derivatives of velocity but second order in time derivatives of the shape function, and so the data that determines a solution of these equations is initial shape of the membrane and its first time derivative together with the value of the velocity field at any instant of time.

The equations (1.7) are the main result of this paper. These equations are completely new and capture the nonlinear dynamics of the slow quasi normal modes of EST and collaborators (which previously had only been understood at the linearized level). We have learnt that the authors of the upcoming paper [18] have independently obtained a set of nonlinear equations that describe static large $d$ membranes. While the membrane equations (1.7) of this paper apply to aribitrary dynamical configurations, it should be possible to specialize these equations and thereby rederive the results of [18] (at leading order in $1 / d$ ) as a special case; we leave this exercise to future work.

It has, of course, been previously noticed that the dynamics of black hole horizons resemble the equations of hydrodynamics on a membrane. The fact that the dynamics of black hole horizons is governed at large $d$ by the precise equations (1.7) can be regarded as a mathematical realization of this idea in an appropriate context. In the title of this paper we have used the phrase 'membrane paradigm' to summarize our conclusion that black hole dynamics is precisely captured by an effective theory on a membrane. It would be interesting to investigate the relationship between the equations (1.7) and the work initiated in $[19,20]$ (see also [21] and subsequent work) that is more conventionally referred to as the membrane paradigm.

As a test of our equations we have computed the shape function, normal vector fields, extrinsic curvatures and effective velocity one form fields for Schwarzschild black holes as well as Myers-Perry black holes (with rotations in upto $\left[\frac{p+1}{2}\right]$ planes) at large $D$, and have explicitly verified that they obey the equations (1.7). We have also linearized (1.7) about the Schwarzschild solutions and computed the spectrum of small fluctuations; our results perfectly match the spectrum of quasinormal modes computed in $[8] .^{5}$

The reader might wonder how it is consistent to have a membrane fluctuating around in a theory of gravity without emitting gravitational radiation. We believe that the answer

\footnotetext{
${ }^{5}$ Note that the quasinormal frequencies have imaginary pieces at the leading nontrivial order in the $\frac{1}{d}$ expansion. It follows that the equation (1.7) are inherently dissipative, in contrast to the equations of hydrodynamics which are non dissipative at leading (perfect fluid) order.
} 
to this question is that our membrane does emit radiation, but that the amplitude of the radiation is of order $e^{-d}$ any finite distance away from the membrane. The radiation may presumably be regarded as resulting from an effective coupling of the form

$$
\int d^{p+3} x T_{\mu \nu} h^{\mu \nu}
$$

Here $h$ is the metric fluctuation to the exterior of the membrane. $T_{\mu \nu}$ is the effective stress tensor of the membrane which is delta function localized on the membrane surface and must be conserved as a consequence of (1.7). It would be very interesting to derive this structure (and in particular the formula for $T_{\mu \nu}$ ) in detail. We postpone this task to future work.

The discussion of the previous paragraph would appear to suggest that our membrane loses very little energy to radiation. While this is possible it also possible that the smallness of the radiation field away from the membrane is actually a consequence of the extremely rapid fall off of a finite energy radiation field due to the large $d$ version of the the inverse square law. Restated, the highly dissipative nature of our membrane dynamics may be entirely due to "radiation" into the horizon (as in the case of the fluid-gravity correspondence) but may also be partly due to radiation to infinity. We postpone further discussion of this important issue to future work.

The equations (1.7) are the principal results of this paper. Our equations suggest many interesting questions and generalizations. If the loss of energy to infinity is small, it may be possible to recast (1.7) as the equations of conservation of the membrane stress tensor; this would yield a formulation of membrane dynamics analogous to the usual formulation of hydrodynamics. It should be possible to imitate the analysis of [22] to uplift the area form on the event horizon to an entropy current on the membrane whose divergence is point wise positive for every consistent solution to the equations of motion. It would be interesting to find the first correction in $\frac{1}{d}$ to (1.7) and also to find the analogue of (1.7) in the Einstein-Maxwell system. Finally it would be interesting to study the phenomenology of these equations; for instance to use them to study the head on collision of two spherical black holes. We leave all these questions to future work.

\section{Membrane like solutions in the large $d$ limit}

\subsection{Dimensional reduction of $\mathrm{SO}(d+1)$ invariant metrics}

As we have explained in the introduction, in this paper we study the vacuum Einstein equations (1.1), restricting attention to solutions that preserve $\mathrm{SO}(d+1)$ invariance, i.e solutions of the form (1.4). The effective action for the metric $g_{\mu \nu}$ and the scalar field $\phi$ in (1.4) takes the form

$$
S=\int \sqrt{g} e^{\frac{d \phi}{2}}\left(R+d(d-1) e^{-\phi}+\frac{d(d-1)}{4}(\partial \phi)^{2}\right)
$$


The equations of motion that follow from the variation of this action may be shown to take the form (see appendix A)

$$
\begin{aligned}
& e^{-\phi}(d-1)-\frac{d}{4}(\partial \phi)^{2}-\frac{1}{2} \nabla^{2} \phi=0 \\
& R_{\mu \nu}=\frac{d}{2} \nabla_{\mu} \nabla_{\nu} \phi+\frac{d}{4} \nabla_{\mu} \phi \nabla_{\nu} \phi
\end{aligned}
$$

\section{$2.2 \quad$ A large $d$ limit}

We are interested in (2.2) in the large $d$ limit. A glance at (2.2) shows that $\phi$ is not on the same footing as $g_{\mu \nu}$ in this limit; derivatives of $\phi$ are weighted with an additional factor of $d$ compared to derivatives of the metric $g_{\mu \nu}{ }^{6,7}$ It follows that nontrivial solutions in the large $d$ limit must involve two length scales; a short length scale of order $\frac{1}{d}$ for the variations of the metric $g_{\mu \nu}$ and a longer length scale of order unity for the variations of $\phi .^{8}$ It is thus possible - and very natural - to choose $x^{\mu}$ coordinates for the equations in which the metric and $\phi$ are both of order unity, but derivatives of $\phi$ are of order unity while those of the metric are of order $d$. We choose to employ such a coordinate system in what follows.

How can we describe a metric which varies on the length scale $\frac{1}{d}$ in the large $d$ limit, and yet is nontrivial over macroscopic length scales? We employ the following strategy. Choose a particular point $x_{0}^{\mu}$ and blow up a region of size $\frac{1}{d}$ about this point to unit size and then appropriately rescale the metric and dilaton gradient. In equations ${ }^{9}$

$$
\begin{aligned}
x^{\mu} & =x_{0}^{\mu}+\alpha_{a}^{\mu} \frac{y^{a}}{d} \\
G_{a b} & \equiv d^{2} g_{a b} ; \quad g_{\mu \nu}=d^{2} \alpha_{\mu}^{a} \alpha_{\nu}^{b} g_{a b}=\alpha_{\mu}^{a} \alpha_{\nu}^{b} G_{a b} \\
\chi_{a} & \equiv \nabla_{a} \phi \times d=\alpha_{a}^{\mu} \nabla_{\mu} \phi
\end{aligned}
$$

We must then repeat the procedure of this paragraph for many $x_{0}^{\mu}$ (so that the union of the patches about each of these points is the full manifold) and make sure that descriptions in distinct patches agree where they overlap.

Focussing on the patch around $x_{0}^{\mu},(2.2)$ may be rewritten as

$$
\begin{aligned}
\frac{1}{2} \nabla_{a} \chi^{a} & =e^{-\phi} \frac{d-1}{d}-\frac{1}{4} \chi^{2} \\
R_{a b} & =\frac{1}{2} \nabla_{a} \chi_{b}+\frac{1}{4 d} \chi_{a} \chi_{b}
\end{aligned}
$$

where (2.4) is written regarding $G_{a b}$ as the metric (curvatures, Christoffel symbols and covariant derivatives are also constructed out of $G_{a b}$.)

\footnotetext{
${ }^{6}$ Recall that $d \nabla_{\mu} \nabla_{\nu} \phi$ has a term involving one derivative and one Christoffel symbol, and so should be thought of as having one $\phi$ derivative and one metric derivative at leading order.

${ }^{7}$ The intuitive reason for this difference is clear; $\phi$ controls the size of a $d$ sphere. In the large $d$ limit we should thus expect derivatives of $\phi$ to be more 'expensive' than those of the metric.

$8 \frac{1}{\sqrt{(\nabla \phi)^{2}}}$ gives a covariant estimate for the longer length scale; the square of the Riemann tensor gives a covariant estimate for the inverse fourth power of the shorter length scale.

${ }^{9}$ We rescale $\nabla \phi$ in order to account for the extra factor of $d$ in derivatives of $\phi$ as compared to derivatives of the metric.
} 
At leading order (2.4) reduces to ${ }^{10}$

$$
\begin{aligned}
-2 G^{a b} \Gamma_{a b}^{c} \chi_{c} & =4 e^{-\phi}-\chi^{2} \\
2 R_{a b} & =-\Gamma_{a b}^{c} \chi_{c}
\end{aligned}
$$

where $\phi$ and $\chi_{a}$ are constants. Note that $d$ has disappeared from the leading order equations (2.5).

\subsection{Flat solutions of the leading large $d$ equations}

It is obvious that constant $G_{a b}$ is a solution to (2.5) provided

$$
e^{\phi} \chi^{2}=4
$$

(here $\left.\chi^{2}=G^{a b} \chi_{a} \chi_{b}\right)(2.6)$ is equivalent to (see (2.3))

$$
e^{\phi}\left(g^{\mu \nu} \partial_{\mu} \phi \partial_{\nu} \phi\right)=4
$$

Its easy to check that (2.7) is satisfied at every point in (global) flat space. In the coordinate system of (1.3) this follows upon noting that $\phi=2 \ln S$ and $e^{\phi}=S^{2}$.

\subsection{The black brane}

In this subsection we present a less trivial class of exact solutions to the leading order large $d$ equations of motion (2.5). The solutions we present in this subsection maintain translational invariance in $p+2$ out of the $p+3$ dimensions in which we work; we refer to them as black brane solutions (in analogy with the black branes of asymptotically AdS Einstein gravity with a negative cosmological constant).

Let $G_{F}$ denote any metric whose components, $\left(G_{F}\right)_{a b}$ are all constants. Consider the metric

$$
G_{F}+e^{-R} O^{2}
$$

where $R$ is a coordinate ( $d R$ is the direction in which translational invariance is broken) and $O$ is a constant one form (i.e. each component of $O_{\mu}$ is a constant). It turns out that (2.8) is an exact solution to the equation of motion (2.5) provided that

$$
e^{\phi} \chi \cdot \chi=4, \quad(2 d R-\chi) \cdot d R=0, \quad(2 d R-\chi) \cdot O=0, \quad O \cdot O=0
$$

(all dot products in equation (2.9) are evaluated using the metric $\left.G_{F}\right) .{ }^{11}$ In appendix A.2 we have demonstrated that it is possible to choose coordinates in which the metric (2.8) takes the form

$$
\begin{aligned}
e^{-\phi} & =\frac{1}{x_{0}^{2}}, \quad \chi=2 d R+\frac{2}{x_{0}} d X \\
d s^{2} & =2 d R d V-a\left(1-e^{-R}\right) d V^{2}+d Y^{i} d Y^{i}+\frac{d X^{2}}{1-a x_{0}^{2}}
\end{aligned}
$$

\footnotetext{
${ }^{10}$ Note that$$
-\Gamma_{c d}^{b} G^{c d} \equiv \frac{1}{\sqrt{G}} \partial_{a}\left(G^{a b} \sqrt{G}\right)
$$

${ }^{11}$ Note that the metric (2.8) does not provide a completely unambiguous definition of the coordinate $R$ and the one form $O$. The redefinition $R \rightarrow R+m$ and $O \rightarrow e^{\frac{m}{2}} O$ leaves (2.8) unchanged. It follows that the one form $O$ and the coordinate $R$ are defined only upto this ambiguity.
} 
At fixed $x_{0}$ it follows that (2.8) and (2.9) define a one parameter set of metrics upto coordinate transformations. In appendix A.2 we demonstrate that the one parameter, $a$ in $(2.10)$, is a consequence of the scaling symmetry ${ }^{12}$ of the vacuum Einstein equations without a cosmological constant.

The black brane solutions presented in this subsection are not really new. They can be obtained from the well known Schwarzschild solution (1.2) as follows. We choose any point on the horizon of the black hole, and then zoom into a patch of size $\frac{1}{d}$ of that point by performing the coordinate changes (2.3). The resultant metric turns out to be the black brane; the parameter $a$ of the black brane is a function of $r_{0}$ of the original black hole. In other words the black brane solutions of this section are simply large $d$ limits of appropriately 'boosted' versions of the 'near horizon' solutions of Schwarzschild black holes (of various sizes).

\subsection{Sewing black branes into a membrane}

In this subsection we will present the construction of a class of metrics with several interesting properties. We start with flat space and perturb the metric in a manner controlled by a scalar function $B$ and a null vector field $O$.

Let $B$ be an $\mathrm{SO}(d+1)$ invariant scalar function that lives on the flat space (1.3). $\mathrm{SO}(d+1)$ invariance ensures that $B$ is a function only of the coordinates $\left(w^{a}, S\right)$ (see around (1.3) for definitions). We choose the function $B$ so that its zeroes form a codimension one closed surface -for instance of the topology of a sphere times time - in the flat spacetime (1.3). We refer to this surface as the membrane. We require that $B$ have only simple zeroes. The zeroes of $B$ divide flat space up into two disjoint regions; we refer to the part of spacetime that includes infinity as the outside, and the other region as the inside. We require that $B$ is negative on the inside and positive on the outside, and also that $d B . d S>0$.

In terms of $B$ we define an auxiliary scalar function $\psi$ by the equation

$$
\psi=1+\frac{d B \cdot d \phi}{2 d B \cdot d B} B
$$

It follows from the conditions on $B$ that $\psi<1$ inside, that $\psi=1$ on the membrane and that $\psi>1$ on the outside.

In addition to $B$, let us consider a null $\mathrm{SO}(d+1)$ invariant one form field $O$ which lives in flat space. As $O$ is null, $O . O=0$ everywhere. We also demand that

$$
\left.O \cdot\left(\frac{d \phi}{2}-d \psi\right)\right|_{B=0}=0 .
$$

where (2.12) is required to hold only on the membrane. The functions $B$ and $O$, written as functions of $\left(w^{a}, S\right)$ and $\left(d w^{a}, d S\right)$, are assumed to be independent of $d$.

Now consider the spacetime

$$
d s^{2}=d s_{\text {flat }}^{2}+\frac{O_{\mu} O_{\nu} d x^{\mu} d x^{\nu}}{\psi^{d+p}}
$$

\footnotetext{
${ }^{12}$ Namely that uniform scaling of the metric by a constant maps solutions to solutions.
} 
Here $d s_{\text {flat }}^{2}$ is the metric of flat space (for instance $d s_{\text {flat }}^{2}$ is given by (1.3) in a useful coordinate system).

The spacetime (2.13) has several interesting properties. First, it approaches the metric of flat space exponentially rapidly in the outside region. The deviation of (2.13) from flat space scales like $e^{-(\psi-1) d}$. It follows, in particular, that (2.13) solves Einstein's equations at large $d$ at any point outside the membrane such that $\lim _{d \rightarrow \infty}(\psi-1) d=\infty$.

The only points outside the membrane at which it is not obvious that (2.13) solves Einstein's equations is points for which $\psi-1$ is scaled to be of order $\frac{1}{d}$ as $d$ is taken to infinity. Recall that $\psi=1$ defines the membrane surface. As $\psi$ is a smooth function in the neighbourhood of the membrane, all such points lie within a distance $\frac{1}{d}$ of the membrane. We refer to this neighbourhood of the membrane as the 'membrane region'. We will now argue that (2.13) solves the leading order large $d$ Einstein equations everywhere within the membrane region.

In the membrane region $\psi^{p+d} \sim e^{(p+d)(\psi-1)}$. As $\psi$ is a smooth regular $d$ independent function in the neighbourhood of the membrane, it follows that $e^{(p+d)(\psi-1)}$, and so the metric (2.13), varies over a length scale of order $\frac{1}{d}$. In contrast $\phi=2 \ln S$ varies over the length scale unity.

Let us now study the metric in the membrane region in more detail, using the strategy outlined around (2.3). For this purpose it is useful to use $\psi-1$ as one of our original coordinates, and choose $R=d(\psi-1)$ as the corresponding rescaled coordinate for the blown up analysis of (2.3). Choosing $x_{0}^{\mu}$ in (2.3) to be any point on the membrane, it is easily seen that (2.13) reduces precisely to the black brane metric (2.8) at leading order in the large $d$ limit. As the flat space metric and $O$ were smooth functions in the original coordinates, they are constants after blowing up (to leading order in $\frac{1}{d}$ ) exactly as for (2.8). Moreover all the conditions (2.9) are also easily seen to be obeyed. The first of (2.9) follows from the fact that $\phi=2 \ln S$. The second equation follows upon using (2.11) and the fact that $B$ vanishes on the membrane. The third of (2.9) follows directly from (2.12) and the fourth of (2.9) follows form the fact that $O$ is a null field.

Finally, in appendix A.3 we demonstrate that the event horizon of the metric (2.13) lies within the membrane region. For this reason we do not care whether (2.13) obeys Einstein's equations inside the membrane (i.e. for $1-\psi \gg \frac{1}{d}$ ) as this region of spacetime is causally disconnected from the outside.

In summary the metric (2.13) obeys the leading large $d$ Einstein equations everywhere outside its event horizon. This fact allows us to use (2.13) as the starting point for the construction of solutions to Einstein's equations in a systematic expansion in $\frac{1}{d}$.

\subsection{Equivalent starting points for perturbation theory and a 'gauge choice'}

Recall that the metric (2.13) obeys Einstein's equations only at leading order in large $d$. If two metrics of the form (2.13) coincide at leading order in large $d$ (but differ at subleading order) they should be regarded as equivalent starting points for perturbation theory. With this definition of equivalence classes we show in appendix A.4 that inequivalent classes of metrics (2.13) are labelled by the shape of the membrane and the value of the one form 
field $O$ on the membrane. In other words functions $B$ and one forms $O$ related by

$$
O \rightarrow O+\delta O, \quad B \rightarrow \alpha B
$$

are equivalent starting points for perturbation theory. ${ }^{13}$

In order to proceed with our perturbative construction of metrics in the large $d$ limit, we will find it useful to choose one representative in each equivalence class; we do this by imposing the following 'gauge' conditions on $B$ and $O$

$$
\begin{aligned}
(\partial B . \nabla) \nabla_{\mu} B & =0 \\
\partial B . \nabla O & =0
\end{aligned}
$$

The first condition in equation (2.15) asserts that $\nabla_{\mu} B$ obeys the geodesic equation with affine parametrization. The second condition in (2.15) asserts that $O$ is simply parallel transported along these geodesics.

It is useful to note a simple consequence of (2.15)

$$
\nabla_{\alpha}\left(\partial_{\mu} B \partial^{\mu} B\right)=2 \partial^{\mu} B \nabla_{\mu}\left(\nabla_{\alpha} B\right)=0 .
$$

In other words the norm of the one form $d B$ is a constant - not just along the geodesics, but everywhere in space. An overall scaling of $B$ can then be used to set the norm of this one form to unity. With this choice of scaling, $d B$ is, in particular, the unit normal one form to the membrane. We employ this choice in what follows. ${ }^{14}$ In the rest of this paper we use the notation

$$
n_{\mu}(x)=\partial_{\mu} B
$$

which highlights the fact that $\partial_{\mu} B$ is the unit normal vector on the membrane. In this new notation (2.15) become

$$
n . \nabla n=n . \nabla O=0
$$

Finally let us turn to a more explicit parametrization of the one form $O$. Let

$$
O=e^{h}\left(1,-u_{a}\right)
$$

(where the first component in $\left(1,-u_{a}\right)$ is the coefficient of $d S$, and the remaining components refer to the components of $d w^{a}$. It follows from the fact that $O$ is null that

$$
u^{2}=-1
$$

Moreover, the second of (2.15) implies that

$$
n . \nabla u_{a}=n . \nabla h=0
$$

\footnotetext{
${ }^{13}$ We assume $\delta O$ vanishes on the membrane while $\alpha$ is nonzero but finite on the membrane.

${ }^{14}$ With these conventions there is an elegant geometrical construction of the function $B$ in the neighbourhood of the membrane. Let us foliate spacetime with geodesics that pierce the membrane in a normal direction. The value of $B$ at any point $P$ is simply equal to the signed length along the appropriate geodesic from the membrane to $P$.
} 
Finally recall that $O \cdot\left(d \psi-\frac{d S}{S}\right)=0$ on the membrane. Using $\partial_{\mu} \psi=\frac{\partial_{S} B \partial_{\mu} B}{S}$ on the membrane, this condition reduces on the membrane to

$$
u . \partial B=\partial_{S} B-\frac{1}{\partial_{S} B}
$$

Now it is easily verified that the $\nabla^{\mu} B \nabla_{\mu}$ derivative of the equation (2.22) vanishes (this follows from the second of (2.15)) implying that (2.22) holds everywhere, and not just on the membrane.

\section{Correction at first order in $\frac{1}{d}$}

In this section we will improve the metric (2.13) to ensure that it solves Einstein's equations at first subleading order in $\frac{1}{d}$. Our strategy to do this is straightforward. We will expand the metric (2.13) about $x_{0}^{\mu}$ in the patch coordinates (2.3), but will keep both the leading and first subleading terms in the $\frac{1}{d}$ expansion. In an appropriate choice of patch coordinates the metric reduces to the black brane solution (2.10) corrected by terms of order $\frac{1}{d}$ that are linear in $\delta x^{\mu}=x^{\mu}-x_{0}^{\mu}$. This metric fails to solve Einstein's equations at first subleading order in $\frac{1}{d}$. In order to fix this defect we allow the metric (2.13) to be corrected at first order in $\frac{1}{d}$ and determine the form of the correction that ensures that Einstein's equations are obeyed at first order in $\frac{1}{d}$. As we will see below, the correction is nonsingular if and only if the membrane shape and $u_{\mu}$ field obey the equations of motion (1.7).

\subsection{Interpretation of Taylor coefficients and constraints}

The functions $B(x), h(x)$ and $u_{\mu}(x)$ can be Taylor expanded about any point $x_{0}^{\mu}$ as follows

$$
\begin{aligned}
B(x) & =B\left(x_{0}\right)+\nabla_{\mu} B\left(x_{0}\right) \delta x^{\mu}+\nabla_{\mu} \nabla_{\nu} B\left(x_{0}\right) \frac{\delta x^{\mu} \delta x^{\nu}}{2}+\ldots \\
h(x) & =h\left(x_{0}\right)+\nabla_{\mu} h\left(x_{0}\right) \delta x^{\mu}+\ldots \\
u_{\mu}(x) & =u_{\mu}\left(x_{0}\right)+\nabla_{\nu} u_{\mu}\left(x_{0}\right) \delta x^{\nu}+\ldots
\end{aligned}
$$

where $\delta x^{\mu}=x^{\mu}-x_{0}^{\mu}$. Let us define the functions

$$
\nabla_{\mu} B(x)=n_{\mu}(x), \quad \nabla_{\mu} \nabla_{\nu} B(x)=K_{\mu \nu}(x), \quad \nabla_{\mu} h(x)=h_{\mu}(x), \quad \nabla_{\nu} u_{\mu}(x)=u_{\mu \nu}(x)
$$

It follows that the Taylor expansion (3.1) may be rewritten as

$$
\begin{aligned}
B(x) & =B\left(x_{0}\right)+n_{\mu}\left(x_{0}\right) \delta x^{\mu}+K_{\mu \nu} \frac{\delta x^{\mu} \delta x^{\nu}}{2}+\ldots \\
h(x) & =h\left(x_{0}\right)+h_{\mu}\left(x_{0}\right) \delta x^{\mu}+\ldots \\
u_{\mu}(x) & =u_{\mu}\left(x_{0}\right)+u_{\mu \nu}\left(x_{0}\right) \delta x^{\nu}+\ldots \\
n_{\mu}(x) & =n_{\mu}\left(x_{0}\right)+K_{\mu \nu}\left(x_{0}\right) \delta x^{\nu}+\ldots
\end{aligned}
$$

Recall that the norm of $n_{\mu}(x)$ is a constant (see (2.16)) and that we have chosen to normalize $B$ to set this constant to unity. It follows from the first of $(2.15)$ that

$$
n^{\mu}(x) K_{\mu \nu}(x)=0
$$

Using (3.1) and (3.4), it follows that the extrinsic curvature at any point $x_{0}^{\mu}$ on the membrane surface is given by simply $K_{\mu \nu}\left(x_{0}\right)$. 
The derivatives of the velocity field are not all independent; differentiating (2.22) we find

$$
n^{\mu} u_{\mu \nu}=\left(-K_{\nu \mu} u^{\mu}+K_{\nu s}+\frac{1}{n_{s}^{2}} K_{\nu s}\right)
$$

and differentiating (2.20) we have

$$
u^{\mu} u_{\mu \nu}=0
$$

\subsection{Data}

At any point on the membrane, there is a $p$ dimensional subspace orthogonal to the three dimensional subspace spanned by $n_{\mu}, u_{\mu}, d S$. In this brief subsection we list a basis of independent Taylor expansion coefficients, labelled by their transformation properties under the local tangent space $\mathrm{SO}(p)$ group. Recall that the Taylor expansion coefficients $K_{\mu \nu}$, $u_{\mu \nu}$ and $h_{\mu}$ are not independent as they are constrained by (3.4), (3.6), (2.21) and (3.5); these equations can be used to determine all the 'other' expansion coefficients in terms of the independent coefficients listed below

- Scalars:

$$
\begin{aligned}
& S_{1}=K_{s s}, \quad S_{2}=u^{\mu} K_{s \mu}, \quad S_{3}=u^{\mu} u^{\nu} K_{\mu \nu}, \quad S_{4}=\mathcal{P}^{\mu \nu} K_{\mu \nu} \\
& S_{5}=h_{s}, \quad S_{6}=u^{\mu} h_{\mu}, \quad S_{7}=\mathcal{P}^{\mu \nu} u_{\mu \nu}
\end{aligned}
$$

- Vectors:

$$
V_{1}^{\mu}=\mathcal{P}^{\mu \alpha} u_{\alpha s}, V_{2}^{\mu}=\mathcal{P}^{\mu \alpha} u^{\nu} u_{\alpha \nu}, V_{3}^{\mu}=\mathcal{P}^{\mu \alpha} K_{s \alpha}, V_{4}^{\mu}=\mathcal{P}^{\mu \alpha} u^{\nu} K_{\nu \alpha}, V_{5}^{\mu}=\mathcal{P}^{\mu \alpha} h_{\alpha}
$$

- Symmetric Tensor:

$$
T_{1}^{\mu \nu}=\mathcal{P}^{\mu \alpha} P^{\mu \beta}\left[K_{\alpha \beta}-\frac{\eta_{\alpha \beta}}{p} \mathcal{P}^{\theta \phi} K_{\theta \phi}\right], T_{2}^{\mu \nu}=\mathcal{P}^{\mu \alpha} P^{\mu \beta}\left[\frac{u_{\alpha \beta}+u_{\beta \alpha}}{2}-\frac{\eta_{\alpha \beta}}{p}\left(\mathcal{P}^{\theta \phi} u_{\theta \phi}\right)\right]
$$

- Anti-symmetric tensor:

$$
A^{\mu \nu}=\mathcal{P}^{\mu \alpha} P^{\nu \beta}\left[\frac{u_{\alpha \beta}-u_{\beta \alpha}}{2}\right]
$$

As we have explained above, our 'gauge' condition (2.15) ensures that normal derivatives of $h$ and the velocity field vanish (see (2.21)). This ensures that the derivatives of these quantities are equal to derivatives projected to the world volume of the membrane, i.e.

$$
u . \nabla=u . \nabla-(n . u) n . \nabla, \quad \partial_{S}=\partial_{S}-n_{S}(n . \nabla)
$$

As the $h$ and velocity fields are physical only on the surface of the membrane, all derivatives in the data above (and in the final first order metric for our black hole below) should actually be thought of as projected derivatives. With this replacement our final results for the metric and equation of motion apply without reference to the arbitrary gauge choice (2.15).

\subsection{Structure of the first correction to the metric}

As mentioned above, we will need to correct the metric (2.13) in order to ensure that Einstein's equations are obeyed at first subleading order in $\frac{1}{d}$. We adopt $S$, the size of the $d$ sphere as one of our coordinates. This choice leaves us with $p+2$ remaining coordinate freedoms. We fix this freedom in the following manner. Let $d \psi, d S-S d \psi, O$ and $\mathcal{P}_{\nu}^{\mu} d x^{\nu}$ form a basis of the $p+3$ dimensional space of one forms at any point. The correction metric 
can be regarded, point wise, as a symmetric quadratic form of these one forms. Our choice of gauge is that the only terms involving $d \psi$ in the correction metric are of the form ${ }^{15}$

$$
d \psi \otimes\left(d \psi-\frac{d S}{S}\right)
$$

The correction metric will not contain terms proportional to $d \psi \otimes d \psi, d \psi \otimes O$ and $d \psi \otimes \mathcal{P}_{\nu}^{\mu} d x^{\nu}$.

With this choice of gauge the metric (2.13), corrected to first order in $\frac{1}{d}$, must take the form

$$
\begin{aligned}
d s^{2}= & \eta_{\mu \nu} d x^{\mu} d x^{\nu}+\psi^{-(d+p)} O^{2} \\
& +\frac{\psi^{-d}}{d}\left[K_{1}\left(x^{\alpha}\right) O^{2}+2 K_{2}\left(x^{\alpha}\right)(d S-S d \psi) \otimes O\right. \\
& +K_{3}\left(x^{\alpha}\right)(d S-S d \psi)^{2}+2 K_{4}\left(x^{\alpha}\right) d \psi \otimes(d S-S d \psi) \\
& +2 Q_{1}^{\beta}\left(x^{\alpha}\right) O_{\mu} \mathcal{P}_{\nu}^{\beta} d x^{\mu} d x^{\nu}+2 Q_{2}^{\beta}\left(x^{\alpha}\right) \mathcal{P}_{\nu}^{\beta} d x^{\nu}(d S-S d \psi) \\
& \left.+\mathcal{T}_{\alpha \beta}\left(x^{\alpha}\right) \mathcal{P}_{\mu}^{\alpha} \mathcal{P}_{\nu}^{\beta} d x^{\mu} d x^{\nu}\right]+\mathcal{O}\left(\frac{1}{d}\right)^{2} .
\end{aligned}
$$

The $\mathcal{O}\left(\frac{1}{d}\right)$ correction piece in (3.8) has been added to ensure that (3.8) solves Einstein's equations at first subleading order in $\frac{1}{d}$. This correction is needed because the first line of (3.8) does not, by itself, have this property. Let us recall why this is the case. Upon expanding the first line of (3.8) in a patch to first subleading order in $\frac{1}{d}$, we find the black brane background corrected by $\mathcal{O}(1 / d)$ fluctuations. Now the fluctuations - which are all proportional to the data of the previous subsection plus a constant piece $^{16}$ - fail to solve the leading order Einstein equations, giving rise to sources proportional to first order data. Moreover the black brane metric itself is a solution only to the leading order Einstein equations, and so yields a constant source at order $\frac{1}{d}$ when plugged into the exact Einstein equations. As the correction part of the metric in (3.8) is chosen to cancel these sources, it follows that the unknown functions in (3.8) must take the form ${ }^{17}$

$$
\begin{aligned}
K_{a}\left(x^{\alpha}\right) & =\sum_{A=0}^{7} K_{a}^{A}(R) S_{A}, \quad a=\{1,2,3,4\}, \quad S_{0}=1 \\
Q_{a}^{\mu}\left(x^{\alpha}\right) & =\sum_{A=1}^{5} Q_{a}^{A}(R) V_{A}^{\mu}, \quad a=\{1,2\} \\
\mathcal{T}^{\mu \nu}\left(x^{\alpha}\right) & =\sum_{A=1}^{2} \mathcal{T}_{A}(R) T_{A}^{\mu \nu} \\
\text { where } \quad R & =d(\psi-1)
\end{aligned}
$$

\footnotetext{
${ }^{15}$ We use the symbol $\otimes$ to denote the symmetrized product of two one forms. If $A=A_{\mu} d x^{\mu}$ and $B=B_{\mu} d x^{\mu}$ then $A \otimes B=A_{\mu} B_{\nu} d x^{\mu} d x^{\nu}$. In our convention $A^{2}=A \otimes A$.

${ }^{16}$ The constant arises from the fact that $\frac{1}{\psi^{p+d}}$ reduces to $e^{-R}$ only at leading order at large $d$; there are corrections to this formula at first subleading order in $\frac{1}{d}$.

${ }^{17}$ The appearance of $S_{0}=1$ is a consequence of the fact that we have constant sources (not proportional to the first order data of the previous subsection) as explained above.
} 
The unknown functions $K_{a}^{A}(R), Q_{a}^{A}(R), \mathcal{T}_{A}(R)$ are now functions only of $R$ and can be regarded as constants in the other black brane directions within a patch (their variation in these directions affects the metric only at $\left.\mathcal{O}\left(1 / d^{2}\right)\right)$.

\subsection{Results}

In order to determine the unknown functions in (3.9) we now expand the metric (3.8) in patch centred about a particular point $x_{0}^{\mu}$ and obtain the patch metric expanded to first order in $\frac{1}{d}$ (including terms that come from the Taylor expansion of the first line). We then move to a convenient set of patch coordinates and plug the resultant metric into Einstein's equations. As we explain in detail in appendix B, we find differential equations for all unknown functions in (3.9) which we proceed to solve. The solution to these equations turns out to include a singularity within the membrane region (see appendix B) unless the equations (1.7) are obeyed. When those equations are obeyed the unknown functions in (3.9) are all quite simple and we find ${ }^{18}$ that the final metric ((3.8) with explicit solutions for the functions in (3.9)) is given by

$$
\begin{aligned}
d s^{2}= & \eta_{\mu \nu} d x^{\mu} d x^{\nu}+\psi^{-(d+p)}\left(O_{\mu} d x^{\mu}\right)^{2} \\
& +\frac{\psi^{-d}}{d}\left(O_{\mu} d x^{\mu}\right)\left[K_{1}\left(x^{\alpha}\right)\left(O_{\nu} d x^{\nu}\right)+2 K_{2}\left(x^{\alpha}\right)(d S-S d \psi)-K_{V}\left(x^{\alpha}\right)\left(\mathcal{P}_{\nu}^{\beta} u_{\beta s}\right) d x^{\nu}\right] \\
& +\mathcal{O}\left(\frac{1}{d}\right)^{2}
\end{aligned}
$$

where

$$
\begin{aligned}
K_{1}\left(x^{\alpha}\right)= & -\left[\frac{-c^{6}+11 c^{4}-23 c^{2}+11}{\left.c^{2}-2\right)^{2}\left(c^{2}-1\right)}\right] R-\left[\frac{\left(c^{2}-1\right)\left(3 c^{2}-8\right)}{2\left(c^{2}-2\right)^{2}}\right] R^{2} \\
& +\left[\frac{c^{2} R^{2}+2\left(-4 c^{4}+7 c^{2}+1\right) R}{2 c^{2}\left(2-c^{2}\right)^{2}}\right]\left(u^{\alpha} u^{\beta} K_{\alpha \beta}\right)\left(S n_{S}\right) \\
& -\left[\frac{c^{2} R^{2}+\left(-4 c^{4}+6 c^{2}+2\right) R}{c^{2}\left(1-c^{2}\right)\left(2-c^{2}\right)}\right]\left(u^{\beta} K_{S \beta}\right)\left(S n_{S}\right) \\
& +\frac{R}{1-c^{2}}\left[2 S \partial_{S} h-\left(\mathcal{P}^{\mu \nu} K_{\mu \nu}\right)\left(S n_{S}\right)\right]
\end{aligned}
$$

\footnotetext{
${ }^{18}$ In this paper we have performed all our computations only for the special case $p=2$. The formulae presented in equations (3.11), (3.12) and (3.13) are accurate only for $p=2$. We do not, however, need to perform a new computation in order to obtain the analogues of these three functions at arbitrary $p$. This is because a solution at $p=2$ may be viewed special case of a solution of the membrane equations at all larger values of $p$ - simply by moving some of the $z_{M}$ coordinates into the set of $w_{a}$ coordinates. This operation changes the meaning of $S$, and so induces a non-trivial redefinition of the velocity one form $u_{\mu}$ (recall $u$ is defined so that $O \propto(d S-u)$ ). This requirement of 'redistribution invariance' together with the explicit $p=2$ expressions presented below in (3.11), (3.12) and (3.13) is sufficient to completely fix the three functions $K_{1}, K_{2}$ and $K_{3}$ at all $p$. We postpone the presentation of the explicit expression for the first order correction to the membrane metric at arbitrary values of $p$ to an upcoming paper. Similar logic may be used to constrain the $p$ dependence of the membrane equations of motion (1.7). In this case we find that the requirement of redistribution invariance infact forces the membrane equations to take the form (1.7) at all $p$. This fact will also be explained more fully the upcoming paper mentioned above.
} 


$$
\begin{aligned}
K_{2}\left(x^{\alpha}\right)= & e^{h}\left[\frac{\left(3 c^{4}-6 c^{2}+1\right)+\left(c^{6}-3 c^{4}+2 c^{2}\right) R}{c^{2}\left(c^{2}-2\right)^{2}\left(c^{2}-1\right)}\right] \\
& +e^{h}\left[\frac{\left(3 c^{4}-6 c^{2}+1\right)+\left(c^{2}-2\right) c^{2} R}{c^{4}\left(2-c^{2}\right)^{2}}\right]\left(u^{\alpha} u^{\beta} K_{\alpha \beta}\right)\left(S n_{S}\right) \\
& -e^{h}\left[\frac{-\left(2-c^{2}\right) R+3 c^{2}-5}{c^{2}\left(1-c^{2}\right)\left(2-c^{2}\right)}\right]\left(u^{\beta} K_{S \beta}\right)\left(S n_{S}\right) \\
& -\frac{S e^{h}}{c^{2}\left(1-c^{2}\right)}\left[2\left(2-c^{2}\right)\left(\partial_{S} h\right)-2\left(1-c^{2}\right)\left(u^{\theta} \partial_{\theta} h\right)\right. \\
& \left.-\left(\mathcal{P}^{\mu \nu} K_{\mu \nu}\right)\left(n_{S}\right)+\left(1-c^{2}\right)\left(P^{\mu \nu} u_{\mu \nu}\right)\right] \\
K_{V}\left(x^{\alpha}\right)= & \frac{2 S e^{h}}{\left(1-c^{2}\right)}(1+R) .
\end{aligned}
$$

Here

$$
\begin{aligned}
\mathcal{P}_{\mu \nu} & =\text { The projector perpendicular to } u_{\mu}, n_{\mu} \text { and } d S \\
c^{2} & =1-n_{S}^{2} \\
R & =d(\psi-1)
\end{aligned}
$$

All raising and lowering of the indices have been done using the flat metric $\eta_{\mu \nu}$.

(3.10) is our final result for the metric of membrane spacetimes corrected to first order in $\frac{1}{d}$. We recall that (3.10) solves Einstein's equations to the relevant order if and only if (1.7) are obeyed.

\section{Stationary black hole solutions}

In this section we provide some examples of exact solutions to Einstein's equations that reduce to membrane spacetimes at large $d$. We explicitly verify that these solutions obey the equations (1.7).

\subsection{The Schwarzschild black hole}

The metric for the Schwarzschild black hole of unit radius is given by

$$
d s^{2}=-d t^{2}+d S^{2}+d x_{i} d x^{i}+\left(d t+\frac{x_{i} d x^{i}+S d S}{\sqrt{r^{2}+S^{2}}}\right)^{2} \frac{1}{\left(S^{2}+r^{2}\right)^{\frac{p+d}{2}}}
$$

Hence the zero norm one form $O$ is given by

$$
\begin{aligned}
O & =\frac{S}{\sqrt{S^{2}+r^{2}}}\left(d S-u_{\mu} d x^{\mu}\right) \\
u_{\mu} d x^{\mu} & =-\frac{\sqrt{S^{2}+r^{2}}}{S} d t-\frac{r}{S} d r
\end{aligned}
$$

where $r^{2}=x_{i} x^{i}$. The membrane surface is defined by

$$
S^{2}+x_{i} x^{i}=1
$$


the normal to the surface which has unit magnitude on the surface is given by

$$
n=\frac{S d S+r d r}{\sqrt{S^{2}+r^{2}}}
$$

We see that the conditions $u . u=-1$ and $u . n=n^{s}-\frac{n^{2}}{n^{s}}$ are trivially satisfied.

Let us now focus on the membrane surface $S^{2}+r^{2}=1$. The non-zero components of extrinsic curvature of this surface are obtained as

$$
\begin{aligned}
K_{S S} & =1-S^{2} \\
K_{S i} & =-S x_{i}=K_{i S} \\
K_{i j} & =\delta_{i j}-x_{i} x_{j}
\end{aligned}
$$

We will now check that the Schwarzschild black hole obeys the equations (1.7). The value of the constant $c^{2}$ in these equations turns out to be $r^{2}$ when evaluated on this solution. The various terms appearing in the scalar equations evaluate to

$$
\left(2-c^{2}\right)^{2} K_{S S}=\left(1+S^{2}\right)^{2} r^{2}, \quad\left(1-c^{2}\right)^{2} K_{u u}=S^{4} r^{2}, \quad 2\left(1-c^{2}\right)\left(2-c^{2}\right) K_{S u}=2\left(1-S^{2}\right) S^{2} r^{2}
$$

Plugging all this in the scalar equations of (1.7) we see that it is satisfied. The two terms in the vector equations of (1.7) individually evaluate to zero on the membrane and hence it is also satisfied.

In conclusion, the Schwarzschild black hole spacetime takes the membrane form at large $d$; the shape of the corresponding membrane and its velocity field solve the membrane equations of motion.

\subsection{The Myers-Perry black hole}

Consider the Myers-Perry black hole $[23,24]$ with independent rotations in $q$ distinct two planes. In order to describe this solution within our formalism we need to choose $p+1 \geq 2 q$. We find it convenient to saturate this inequality so that $p+1=2 q$ or $q=\frac{p+1}{2}$.

In appendix A.5 we demonstrate that the Myers-Perry black hole with rotations in $\frac{p+1}{2}$ planes is described by the metric

$$
d s^{2}=-d t^{2}+\sum_{i=1}^{\frac{p+1}{2}}\left(d x_{i}^{2}+d y_{i}^{2}\right)+d S^{2}+\frac{O^{2}}{\rho^{d+p}}
$$

where $S^{2}=z_{M} z^{M}$ as around (1.3) and

$$
\begin{aligned}
& O=A\left(d t+\left(1-\sum_{i=1}^{\frac{p+1}{2}} \frac{\mu_{i}^{2} a_{i}^{2}}{\rho^{2}+a_{i}^{2}}\right) d \rho-\sum_{i=1}^{\frac{p+1}{2}} \frac{a_{i}}{\rho^{2}+a_{i}^{2}}\left(x_{i} d y_{i}-y_{i} d x_{i}\right)\right) \\
& A=\left(m \frac{1}{\prod_{i=1}^{\frac{p+1}{2}}\left(1+\frac{a_{i}^{2}}{\rho^{2}}\right)} \frac{1}{\left(1-\sum_{i=1}^{\frac{p+1}{2}} \frac{a_{i}^{2} \mu_{i}^{2}}{\rho^{2}+a_{i}^{2}}\right)}\right)^{\frac{1}{2}} .
\end{aligned}
$$


and

$$
\begin{aligned}
& \sum_{i=1}^{\frac{p+1}{2}} \frac{x_{i}^{2}+y_{i}^{2}}{\rho^{2}+a_{i}^{2}}+\frac{S^{2}}{\rho^{2}}=1 . \\
& \mu_{i}^{2}=\frac{x_{i}^{2}+y_{i}^{2}}{\rho^{2}+a_{i}^{2}} .
\end{aligned}
$$

so that

$$
\rho d \rho=\frac{1}{\sum_{j=1}^{\frac{p+1}{2}} \frac{x_{j}^{2}+y_{j}^{2}}{\left(\rho^{2}+a_{j}^{2}\right)^{2}}+\frac{S^{2}}{\rho^{4}}}\left(\sum_{i=1}^{\frac{p+1}{2}} \frac{x_{i} d x_{i}+y_{i} d y_{i}}{\rho^{2}+a_{i}^{2}}+\frac{S}{\rho^{2}} d S\right)
$$

The metric (4.7) takes the form (2.13) with $\psi=\rho$ (recall $\rho$ is defined implicitly by (4.9)). It is not difficult to verify that $O_{\mu} O^{\mu}=0$ on the membrane $\rho=1$. It is not difficult to show that

$$
O \propto d S-u_{\mu} d x^{\mu}
$$

where

$$
\begin{aligned}
& u=-\frac{1}{\xi}\left(d t+\sum_{i=1}^{\frac{p+1}{2}}\left(\frac{x_{i}+a_{i} y_{i}}{1+a_{i}^{2}} d x_{i}+\frac{y_{i}-a_{i} x_{i}}{1+a_{i}^{2}} d y_{i}\right)\right) \\
& \xi=1-\sum_{i=1}^{\frac{p+1}{2}}\left(\frac{x_{i}^{2}+y_{i}^{2}}{1+a_{i}^{2}}\right)
\end{aligned}
$$

It is easily verified that $u_{\mu} u^{\mu}=-1$. The unit one form normal to the membrane is proportional to $d \rho$; upon normalizing we find

$$
n=\frac{1}{\sum_{i=1}^{\frac{p+1}{2}} \frac{x_{i}^{2}+y_{i}^{2}}{\left(1+a_{i}^{2}\right)^{2}}+S^{2}}\left(\sum_{i=1}^{\frac{p+1}{2}} \frac{x_{i} d x^{i}+y_{i} d y^{i}}{1+a_{i}^{2}}+S d S\right)
$$

We have explicitly checked that

$$
u . n=n_{S}-\frac{n . n}{n_{S}}
$$

so that (2.22) is obeyed. It follows that the metric (4.7) agrees with our form for the membrane metric at leading order in large $d$.

We have also verified that the expressions (4.11) and (4.12) obey the equations of motion (1.7). In order to verify the scalar equation (first of (1.7)) we explicitly computed the extrinsic curvature tensor. Using our explicit (and lengthy) expressions, we find that the scalar equation (first of (1.7)) is indeed satisfied. 
We have also verified that the vector equation (the second of (1.7) is obeyed. Let us specialize to the case $q=1$ or $p=1$. On the membrane surface we find

$$
\begin{aligned}
\mathcal{P}_{a}^{b}\left(\left(n_{S}^{2}\right)(u . \nabla-(u . n) n . \nabla) u_{b}\right) & =\left(\begin{array}{c}
\frac{a^{2}\left(a^{2}+1\right)\left(x^{2}+y^{2}\right)\left(2 a^{4}-2 a^{2}\left(x^{2}+y^{2}-2\right)-x^{2}-y^{2}+2\right)}{\left(a^{4}-a^{2}\left(x^{2}+y^{2}-2\right)+1\right)^{3}} \\
\frac{a\left(a^{2}+1\right)^{2} y\left(2 a^{4}-2 a^{2}\left(x^{2}+y^{2}-2\right)-x^{2}-y^{2}+2\right)}{\left(a^{4}-a^{2}\left(x^{2}+y^{2}-2\right)+1\right)^{3}} \\
-\frac{a\left(a^{2}+1\right)^{2} x\left(2 a^{4}-2 a^{2}\left(x^{2}+y^{2}-2\right)-x^{2}-y^{2}+2\right)}{\left(a^{4}-a^{2}\left(x^{2}+y^{2}-2\right)+1\right)^{3}}
\end{array}\right) \\
& =\mathcal{P}_{a}^{b}\left(\left(1+n_{S}^{2}\right)\left(\partial_{s}-n_{S} n . \nabla\right) u_{b}\right)
\end{aligned}
$$

(where we have listed the $S, t, x$ and $y$ components of the vector in that order). It follows that the second of (1.7) is satisfied. Note that each individual term in this equations is non-zero; however the two terms cancel between each other. Although we do not explicitly report our results here, we have also verified that the vector equation is satisfied for the case $q=2$ or $p=3$.

\section{Linearization of membrane equations about the Schwarzschild solution}

In the previous section we verified that rotating black holes at large $d$ are solutions to our membrane equations. The equations (1.7) apply to more than stationary configurations; they completely capture time dependent black hole dynamics as well. In this section we apply our equations to a non stationary problem. We determine the most general solution to the equations (1.7) in linearization about the Schwarzschild black hole solution. The solutions we find should map to the most general regular linearized solution of general relativity (with frequencies of order unity) about the Schwarzschild black hole solution, i.e. the 'light' quasinormal modes determined by EST (see the introduction). Indeed we find that the spectrum of the solutions we obtain agrees precisely with the spectrum of the light quasinormal modes of EST to appropriate order in $\frac{1}{d}$. We regard this match as a significant check on our membrane equations (1.7)

In the previous section we demonstrated that the membrane surface

$$
S^{2}+r^{2}=1
$$

(recall $r^{2}=\sum_{i=1}^{p+1} w^{i} w_{i}$ where the index $i$ runs only over spatial coordinates) together with the velocity one form field on the surface of the membrane

$$
u=\left(-\frac{1}{S}\right) d t+\left(-\frac{r}{S}\right) d r
$$

solves the membrane equations (1.7), and that this solution corresponds to a Schwarzschild black hole of unit radius. In order to study small fluctuations about this solution we let the membrane fluctuate, i.e we let our membrane surface be given by

$$
S-\sqrt{1-r^{2}}-a f\left(r, t, \theta^{i}\right)=0
$$


Equivalently, the $S$ coordinate on the membrane is determined in terms of $r, \theta, v$ by the equation

$$
S=\sqrt{1-r^{2}}+a f\left(r, t, \theta^{i}\right)
$$

We also allow the velocity field to fluctuate, i.e we set

$$
u=\left(-\frac{1}{S}+a \delta u_{t}\right) d t+\left(-\frac{r}{S}+a \delta u_{r}\right) d r+a \delta u_{i} d \theta^{i}
$$

where $S$ is given by (5.4). The parameter $a$ in (5.3) and (5.5) measures the amplitude of the perturbation; through this section we work at linear order in $a$.

Our membrane effectively propagates in the $p+3$ dimensional space with metric

$$
d s^{2}=-d t^{2}+d w_{i} d w^{i}+d S^{2}=-d t^{2}+d r^{2}+r^{2} d \Omega_{p}^{2}+d S^{2}
$$

The normal to our perturbed surface is given, to $\mathcal{O}(a)$, by

$$
\begin{aligned}
n= & \left(\sqrt{1-r^{2}}+a r\left(1-r^{2}\right) \partial_{r} f\right) d S-a \sqrt{1-r^{2}} \partial_{t} f d t+\left(r-a \sqrt{\left(1-r^{2}\right)^{3}} \partial_{r} f\right) d r \\
& -a \sqrt{1-r^{2}} \partial_{i} f d \theta^{i}
\end{aligned}
$$

Once again the variable $S$ in (5.7) is given by (5.4). Here $\theta^{i}$ are an arbitrary set of coordinates on the unit $p$ sphere in the metric (5.6), and we have normalized $n$ to ensure that $n . n=1$ upto corrections of order $a^{2}$.

Recall that the velocity field $u$ is constrained to obey $u . u=-1$ and $u . n=n_{S}-\frac{n^{2}}{n_{S}}$. These conditions allow us to solve for $\delta u_{r}$ and $\delta u_{t}$ in terms of $f$; we find

$$
\begin{aligned}
\delta u_{r} & =\frac{a}{r\left(-1+r^{2}\right)}\left(r^{2} f+\left(-1+r^{2}\right)\left(\partial_{t} f+r \partial_{r} f\right)\right) \\
\delta u_{t} & =r \delta u_{r}-a f
\end{aligned}
$$

It follows that we have $p+1$ independent fluctuation fields $\left(f\right.$ and $\left.u_{i}\right)$.

\subsection{The scalar equation}

We find it convenient to use $t, r, \theta^{i}$ as coordinates on the membrane. The dynamics of $f$ is controlled by the scalar equation (the first of (1.7)). Evaluating the extrinsic curvatures associated with the normal vector field (5.7) (we performed the relevant computations on Mathematica), we find that $f$ obeys a linear partial differential equation of second order in derivatives in $t$ and $r$. The equation has no derivatives in the angular coordinates $\theta^{i}$.

It is useful to expand the fluctuation function $f$ as

$$
f\left(r, t, \theta^{i}\right)=\sum_{l m} a_{l m} e^{-i w_{l m} t} G_{l m}(r) Y_{l m}\left(\theta^{i}\right)
$$

Here $l$ and $m$ refer to labels for scalar spherical harmonics of $\mathrm{SO}(p+1) . \quad l$ is a label for the representation of $\mathrm{SO}(p+1),{ }^{19}$ and $m$ is a collective label for the quantum numbers of states within a given representation.

\footnotetext{
${ }^{19}$ If we measure highest weights of representations by eigenvalues under rotations of independent two planes, the representation labelled by $l$ has highest weights $(l, 0,0 \ldots 0)$.
} 
Plugging this expansion into the linear equation for $f$ yields an ordinary differential equation of $G_{l m}(r)$. Because the equation for $f$ had no derivatives in the angular directions, all functions $G_{l m}$ obey the same equation. Making the replacement $G_{l m} \rightarrow G$ and $\omega_{l m} \rightarrow \omega$ we find that the equation takes the form

$$
\begin{aligned}
& -\left(r^{4} w^{2}+w(-2 i+w)+r^{2}\left(1+2 i w-2 w^{2}\right)\right) G(r) \\
& \quad+r\left(-1+r^{2}\right)\left(2\left(r^{2}+i w-i r^{2} w\right) G^{\prime}(r)+r\left(-1+r^{2}\right) G^{\prime \prime}(r)\right)=0
\end{aligned}
$$

where ' denotes derivative w.r.t. $r$. The two linearly independent solutions to the equation (5.10) are given by

$$
G(r)=\frac{1}{\sqrt{i\left(-1+r^{2}\right)(i+4 w)}}\left(C_{1} r^{\frac{1}{2}(1-\sqrt{1-4 i w}+2 i w)}+C_{2} r^{\frac{1}{2}(1+\sqrt{1-4 i w}+2 i w)}\right)
$$

Regularity of these solutions at $r=0$ requires the power of $r$ in (5.11) to be a non negative integer. One of the two solutions above is proportional to $r^{j},{ }^{20}$ provided

$$
w_{S}(j)=-i(j-1) \pm \sqrt{j-1}
$$

While $j$ is required to be a non negative integer this condition is in itself not sufficient to ensure regularity for several different reasons.

Let us first examine the lowest allowed value of $j$, namely $j=0$. At this value of $j$ the two frequencies (5.12) are $w_{S}(0)=0$ and $w_{S}(0)=2 i$. It is easily verified from (5.8) that the mode with $w_{s}(0)=0$ has $\delta u_{r}=0$ and $\delta u_{t}$ regular everywhere. Consequently this mode is a regular small fluctuation of our membrane equations. Its physical interpretation is simple; it corresponds to rescaling the black hole. When, on the other hand, $w_{s}(0)=2 i$, we find from (5.8) that $\delta u_{r} \propto \frac{2}{r \sqrt{1-r^{2}}} \cdot{ }^{21}$ It follows that the velocity field corresponding to this mode is irregular at $r=0$, so this mode is irregular and so unacceptable. This is reassuring as $w=2 i$ would have represented an instability, contradicting the well established stability of Schwarzschild black holes to small fluctuations in all dimensions. In summary, the only allowed frequency at $j=0$ is $w_{S}=0$.

Let us next turn to $j=1$. At this value of $j$ the quadratic equation for $w_{S}(1)$ yields a double zero; both roots in (5.12) are $w=0$. The fact that we find a double root indicates that $j=1$ is a special case that has to be dealt with separately; the two allowed time dependences at this value of $j$ are actually $f$ constant in time and $f \propto t .^{22}$ These two 'zero' modes have a simple physical interpretation. The constant mode corresponds to an infinitesimal translation of the black hole, while the mode linear in time corresponds to an infinitesimal boost for the black hole.

The next issue concerns the angular dependence of modes. Recall that the angular variation of $f$ did not enter our equation, so the reader may at first suspect that we have

\footnotetext{
${ }^{20}$ Which one depends on our precise definition of the square root.

${ }^{21}$ It is easily verified that for $j \geq 1 \delta u_{r}$ is well behaved at $r=0$.

${ }^{22}$ Note that $t=\ln e^{t}$; the linear dependence in time follows from the application of a standard rule. Recall that we plug ansatz $x^{\alpha}$ into a second order linear differential equation and find a double root for $\alpha$ at $\alpha=\alpha_{c}$ then a basis for the solution space of the equation is given by $x_{c}^{\alpha}$ and $x_{c}^{\alpha} \ln x$.
} 
an infinite degeneracy of modes at every $j$, corresponding to the infinite number of possible angular variations, seemingly unconnected with radial behaviour. This is not the case. A scalar function of the form $r^{j} Y_{l m}(\theta)$ is regular at $r=0$ only if i $^{23,24}$

$$
j=l, l+2, l+4 \ldots
$$

It follows that we find a regular solution for $G_{l m}(r)$ provided

$$
w=w_{S}(j), \quad j=l, l+2, l+4 \ldots
$$

In particular the minimum frequency for $G_{l m}(r)$ is $w_{S}(l)$.

In summary the spectrum of frequencies of oscillation in the scalar sector is given by $\omega_{S}(j)$ for $j=0,1,2,3, \ldots$ For any given value of $j$, the modes that have frequency $\omega_{S}(j)$ are scalar spherical harmonics with

$$
l=j, j-2, j-4 \ldots
$$

In other words modes with different angular momenta share the same frequency. Degeneracies are unusual in physics; when they occur they are usually explained by a symmetry. In the current context the relevant symmetry is obvious; fluctuations about the Schwarzschild black hole appear in representations of $\mathrm{SO}(p+d+2)$. The different $\mathrm{SO}(p+1)$ spherical harmonics that descend from a single $\mathrm{SO}(p+d+2)$ spherical harmonic must have the same frequency.

Now consider an $\mathrm{SO}(p+1+d+1)$ spherical harmonic that transforms in the representation $(j, 0,0 \ldots 0)$ (as usual we label representations by the highest weights under rotations in the independent embedding two planes). This representation can be decomposed into a sum over irreducible representations of $\mathrm{SO}(p+1) \times \mathrm{SO}(d+1)$. The number of representations that appear in this decomposition is very large. If we are interested only in those representations that happen to be singlets under $\mathrm{SO}(d+1)$, it is not difficult to convince oneself that the branching rule is

$$
(j, 0,0 \ldots 0) \rightarrow \sum_{m=0}^{\left[\frac{j}{2}\right]}(j-2 m, 0,0 \ldots 0) \otimes I
$$

In other words the set of $\mathrm{SO}(p+1)$ spherical harmonics that have frequencies $\omega_{j}$ are precisely obtained from a single angular momentum $j$ spherical harmonic of $\mathrm{SO}(p+d+2)$ subject to the constraint that we restrict attention only to $\mathrm{SO}(d+1)$ singlets.

In summary, the spectrum of scalar fluctuations is given by $\omega_{S}(j)$ for non negative $j$. Eigenmodes with frequency $\omega_{S}(j)$ are given by the projection onto $\mathrm{SO}(d+1)$ singlets of an angular momentum $j$ spherical harmonic of $\mathrm{SO}(d+p+2)$. This result is in perfect agreement with the frequency of light scalar quasinormal modes about the Schwarzschild black hole obtained by EST in equation (5.29) of [8].

\footnotetext{
${ }^{23}$ The underlying reason for this is that spherical polar coordinates are singular at $r=0$.

${ }^{24}$ This is most easily seen by noting that an $\mathrm{SO}(p+1)$ spherical harmonic of degree $l$ is the restriction of a polynomial of degree $l$ (in the $p+2$ embedding Cartesian variables) to the unit sphere.
} 


\subsection{The vector equation}

In the parametrisation of this subsection the vector equations of (1.7) become

$$
\left(-2+r^{2}\right) u_{i}+r\left(1-r^{2}\right) \partial_{r} u_{i}+\left(1-r^{2}\right) \partial_{t} u_{i}=-\left(1-r^{2}\right) \partial_{i} f
$$

where $u_{i}$ is a one form field that lives on the unit $p$ sphere.

(5.14) is an inhomogeneous or forced linear differential equation in the one form variable $u_{i}$. The solutions of any such equation take the form $H+P$ where $P$ is a particular solution and $H$ is the general solution to the homogeneous part of the equation (i.e. (5.14) with the r.h.s. set to zero).

The particular solution may easily be seen to be regular (the key point here is that the frequencies of the homogeneous part of (5.14), which we work out below, never coincide with the scalar frequencies of the previous subsection) and is of no particular interest to us; we do not bother to work it out. ${ }^{25}$ Our interest is in the spectrum of the homogeneous solutions, to which we now turn.

The most general solution to the homogeneous equation

$$
\left(-2+r^{2}\right) u_{i}+r\left(1-r^{2}\right) \partial_{r} u_{i}+\left(1-r^{2}\right) \partial_{t} u_{i}=0
$$

is easily determined. It is given by a linear combination of terms of the form

$$
u_{i}^{\omega}(r)=e^{-i \omega t} r^{2+i \omega} q_{i}(\theta)
$$

Here $q_{i}(\theta)$ is an arbitrary one form field on the unit sphere.

The solutions above are clearly irregular at $r=0$ unless $2-i \omega$ is a non negative integer. This is a necessary and not a sufficient condition for regularity.

In order to understand the sufficient conditions for regularity more clearly it is useful to decompose

$$
q_{i}=v_{i}+\partial_{i} \lambda, \quad \text { where } \nabla_{i} v^{i}=0
$$

and $\lambda$ is a scalar function on the sphere. $v_{i}$ may then may be decomposed in a basis of vector spherical harmonics, while $\lambda$ may be decomposed in a basis of scalar spherical harmonics.

Let us define the $l^{\text {th }}$ vector spherical harmonic as the set of vector functions on the sphere that transform in the $(l, 1,0, \ldots 0)$ representation of $\mathrm{SO}(p+1)$ (we label representations by eigenvalues of the rotation operators in the independent two planes in the embedding $p+1$ dimensions). It is not difficult to convince oneself that a one form field whose angular part is proportional to the $l_{V}^{\text {th }}$ vector spherical harmonic is regular if and only if the function that multiplies it is a linear combination of terms of the form $r^{j}$ where

$$
j=l_{V}+1, l_{V}+3, l_{V}+5 \ldots
$$

\footnotetext{
${ }^{25}$ It is natural to choose the particular solution of this equation as a linear combination of terms with the scalar frequencies listed in (5.12). At any given frequency, the particular (or forced) solution is proportional to the coefficient of the scalar mode in $f$ at the same frequency, and multiplies a regular function of $r$.
} 
Let us now turn to the regularity of $d \lambda$. As we have already noted in the last subsection, a scalar function $\lambda$ is regular if and only if the coefficient of the $l_{S}^{\text {th }}$ scalar spherical harmonic (defined as the set of functions in the representation $\left(l_{s}, 0,0 \ldots, 0\right)$ of $\mathrm{SO}(p+1)$ ) is a linear sum of terms of the form $r^{j}$ where

$$
j=l_{S}, l_{S}+2, l_{S}+4 \ldots
$$

Notice that for $l_{S}=0 d \lambda=0$ so that the minimum value for $l_{S}$ is unity.

Putting all these facts together, we find that we have regular solutions to the homogeneous vector equation at

$$
\omega=\omega_{V}(j)=-i(j-1), \quad j=1,2 \ldots
$$

This spectrum is in perfect agreement with equation (5.21) of $[8] .{ }^{26}$

Note that $w_{V}$ vanishes at $j=1$. This mode corresponds to turning on an infinitesimal rotation of the black hole (recall that rotations, like angular momenta, transform in the representation $(1,1,0 \ldots 0)$ - i.e. the lowest vector spherical harmonic representation or the appropriate orthogonal groups. All modes with $j \geq 2$ have an imaginary part to their frequency that corresponds to an exponential decay in time. It follows that the Schwarzschild black hole at large $D$ is stable to vector fluctuations (as it was to scalar fluctuations) in accordance with well known results.

\section{Acknowledgments}

We would like to thank P. Ajit, B. Craps, Y. Dandekar, S. Das, R. Emparan, A. Ghosh, R. Gopakumar V. Hubeney, K. Imbasekar, S. Mandal, M. Mandlik, S. Nadkarni, P. Nayak, M. Rangamani, A. Sen, N. Seiberg, S. Trivedi, S. Wadia and A. Yarom for useful discussions. The work of S.B. was supported by an India Israel (ISF/UGC) joint research grant. The work of A.D is supported by an Inspire Fellowship from DST, India. S.M. would like to acknowledge the hospitality of ICTS where this work was intitiated and of the Harish Chandra Research Insitute, IISER Kolkata, the University of Swansea and the Galileo Galilei Institute while this work was in progress. The work of S.M. was supported by an India Israel (ISF/UGC) joint research grant. We would all also like to acknowledge our debt to the people of India for their steady and generous support to research in the basic sciences.

\footnotetext{
${ }^{26}$ We can also list the eigen functions. The allowed values of $j$ for the frequencies of the $l_{v}^{\text {th }}$ vector spherical harmonic are $j=l_{V}, l_{V}+2, l_{V}+4, \ldots$ while the allowed $j$ for the frequencies for the gradient of the $l_{S}^{t h}$ scalar spherical harmonic are $j=l_{s}-1, l_{s}+1, l_{s}+3 \ldots$ We several degeneracies in the frequencies at any particular value of $j$. As in the previous subsection we presume that all representations with a given $\omega_{V}(j)$ have their origin in a single vector spherical harmonic representation of $\mathrm{SO}(d+p+2)$, but we have not carefully verified this claim.
} 


\section{A Technical details}

\section{A.1 Simplification of the dimensionally reduced equations}

Upon varying (2.1) w.r.t. the scalar $\phi$ we find the equation of motion

$$
R+e^{-\phi}(d-2)(d-1)-\frac{d(d-1)}{4}(\partial \phi)^{2}-(d-1) \nabla^{2} \phi=0
$$

Varying w.r.t. $g^{\mu \nu}$ we find

$$
R_{\mu \nu}-\frac{R}{2} g_{\mu \nu}=\frac{d}{2}\left(\nabla_{\mu} \nabla_{\nu} \phi-\nabla^{2} \phi g_{\mu \nu}\right)+\frac{d(d-1)}{2} e^{-\phi} g_{\mu \nu}+\frac{d}{4} \nabla_{\mu} \phi \nabla_{\nu} \phi-\frac{d(d+1)}{8}(\nabla \phi)^{2} g_{\mu \nu}
$$

(A.2) may be somewhat simplified by subtracting half of (A.1) multiplied by $g_{\mu \nu}$ and the equations of motion may more simply be written as

$$
\begin{aligned}
& R+e^{-\phi}(d-2)(d-1)-\frac{d(d-1)}{4}(\partial \phi)^{2}-(d-1) \nabla^{2} \phi=0 \\
& R_{\mu \nu}=\frac{d}{2} \nabla_{\mu} \nabla_{\nu} \phi+\frac{d}{4} \nabla_{\mu} \phi \nabla_{\nu} \phi+g_{\mu \nu}\left((d-1) e^{-\phi}-\frac{d}{4}(\partial \phi)^{2}-\frac{1}{2} \nabla^{2} \phi\right)
\end{aligned}
$$

By subtracting the trace of the second equation from the first we find equivalently

$$
\begin{aligned}
& e^{-\phi}(d-1)-\frac{d}{4}(\partial \phi)^{2}-\frac{1}{2} \nabla^{2} \phi+\frac{p+2}{d-2}\left((d-1) e^{-\phi}-\frac{d}{4}(\partial \phi)^{2}-\frac{1}{2} \nabla^{2} \phi\right)=0 \\
& R_{\mu \nu}=\frac{d}{2} \nabla_{\mu} \nabla_{\nu} \phi+\frac{d}{4} \nabla_{\mu} \phi \nabla_{\nu} \phi+g_{\mu \nu}\left((d-1) e^{-\phi}-\frac{d}{4}(\partial \phi)^{2}-\frac{1}{2} \nabla^{2} \phi\right)
\end{aligned}
$$

where the indices $\mu, \nu$ run over $p+3$ values. Notice that the two terms in the scalar equation are simply proportional to each other, so that the scalar equation is equivalent to

$$
\left((d-1) e^{-\phi}-\frac{d}{4}(\partial \phi)^{2}-\frac{1}{2} \nabla^{2} \phi\right)=0 .
$$

Given this condition, the second term in the vector equation vanishes, and our system of equations reduces to

$$
\begin{aligned}
& e^{-\phi}(d-1)-\frac{d}{4}(\partial \phi)^{2}-\frac{1}{2} \nabla^{2} \phi=0 \\
& R_{\mu \nu}=\frac{d}{2} \nabla_{\mu} \nabla_{\nu} \phi+\frac{d}{4} \nabla_{\mu} \phi \nabla_{\nu} \phi
\end{aligned}
$$

\section{A.2 Details of black branes}

\section{A.2.1 Standard coordinates for the black brane}

In order to verify that (2.8) is indeed a solution, it is useful to move to a convenient coordinate system. Let $x_{0}$ denote the (constant) radius of the $d$ sphere about the point of interest so that

$$
\frac{1}{x_{0}^{2}}=e^{-\phi}=\frac{\chi^{2}}{4}
$$


We then define the coordinate $X$ by the requirement

$$
2 \frac{d X}{x_{0}}=\chi-2 d R
$$

We also define the coordinate $V$ by the requirement that

$$
d V=O
$$

These definitions are sensible because the components of $\chi$ and $O$ are constants. ${ }^{27}$ At this stage we have three special coordinates; $R, X$ and $V$. Let the remaining $p$ coordinates be denoted by $y^{i}$. We employ coordinate redefinitions of the form $y^{i} \rightarrow y^{i}+C_{R}^{i} R+C_{V}^{i} V+C_{X}^{i} X$ to remove all cross terms between $y^{i}$ and $(X, V, R)$. We then employ redefinitions of the form $Y^{i}=\zeta_{j}^{i} y^{j}$ to set the metric in the $Y^{i}$ directions to $\delta_{i j}$. With all these conventions we now proceed to display an explicit form of the metric (2.8).

The choices we have made in the previous paragraph clearly ensure that the metric is a direct sum of $d Y^{i} d Y^{i}$ and a three dimensional metric involving $d X, d V$, and $d R$. We will now determine the form of this three dimensional metric.

The conditions (2.9) ensure that there are no cross terms between $d X$ and either $d V$ or $d R$. It follows that the metric takes the form

$$
\frac{1}{1-a x_{0}^{2}} d X^{2}+b d R^{2}+2 C d R d V-D d V^{2}
$$

where $a, b, C$ and $D$ are arbitrary constants. The fact that $d V$ is null sets $b$ to zero. As we have emphasized in the footnotes above, the coordinates $V$ and $R$ are ambiguous upto a coordinated rescaling and shift. We choose to fix this ambiguity by setting $C=1$. Making this choice, the condition on the squared norm of $\chi$ sets $D=a$, and the metric (2.8) takes the explicit form

$$
\begin{aligned}
e^{-\phi} & =\frac{1}{x_{0}^{2}}, \quad \chi=2 d R+\frac{2}{x_{0}} d X \\
d s^{2} & =2 d R d V-a\left(1-e^{-R}\right) d V^{2}+d Y^{i} d Y^{i}+\frac{d X^{2}}{1-a x_{0}^{2}}
\end{aligned}
$$

We have used an additional shift in $R$ to scale the factor $a$ outside $e^{-R} d V^{2}$ to ensure that the event horizon lies at $R=0$.

\section{A.2.2 Uniform scaling of black branes}

The black brane metric (A.8) appears in a two parameter family labelled by $x_{0}$ and the parameter $a$. There is a simple explanation for the parameter $a$; its existence follows from the fact that Einstein's equations in $p+d+3$ dimensions are invariant under an overall rescaling of the metric. In terms of the reduced fields described above, the map

$$
\left(g_{\mu \nu}, e^{\phi}\right) \rightarrow\left(A g_{\mu \nu}, A e^{\phi}\right)
$$

\footnotetext{
${ }^{27}$ As emphasized in the previous footnote, this definition fixes $V$ only upto a scaling. We will fix the ambiguity of the scale factor below.
} 
takes solutions to solutions. It is easily verified that this rescaling operation does indeed map black branes to black branes. More concretely, the rescaling operation (A.7) applied to the black brane (A.8) yields a new black brane

$$
\begin{aligned}
e^{-\phi} & =\frac{1}{\tilde{x}_{0}^{2}}, \quad \chi=2 d R+\frac{2}{\tilde{x}_{0}} d \tilde{X} \\
d s^{2} & =2 d R d \tilde{V}-\tilde{a}\left(1-e^{-R}\right) d \tilde{V}^{2}+G_{i j} d Y^{i} d Y^{j}+\frac{d \tilde{X}^{2}}{1-a \tilde{x}_{0}^{2}}
\end{aligned}
$$

with

$$
\tilde{a}=\frac{a}{A}, \quad \tilde{x}_{0}^{2}=A x_{0}^{2}, \quad \tilde{V}=A V, \quad \tilde{X}=\sqrt{A} X
$$

The important point here is that this rescaling changes the value of $a$, and so generates black branes with all values of $a$ starting with black branes at $a=1$.

\section{A.3 Location of the event horizon}

We will now demonstrate that the spacetime (2.13) has an event horizon located 'near' to the surface $\psi=1$. As usual we consider a solution of Einstein's equations that eventually settles down into a rotating black hole solution. In this context the event horizon is the unique null manifold that reduces, at large time, to the event horizon of the eventual black hole. In order to compute the event horizon manifold of the spacetime (2.13), let us set up a coordinate system in the neighbourhood of the membrane. Let $\psi$ be one of our coordinates and let $z^{a}$ define the remaining $p+2$ coordinates. Let the event horizon manifold be given by the equation

$$
\psi=\psi_{H}\left(z^{a}\right)
$$

We will now demonstrate that

$$
\psi_{H}=1+\frac{\psi_{1}}{d}+\ldots
$$

The argument is very simple. The event horizon is determined by the requirement that the one form

$$
d \psi+\partial_{a} \psi_{H} d z^{a}
$$

is null. Let us work in a small patch around the point $x_{0}^{\mu}$ which lies on the horizon. We have argued above that the metric is that of the black brane with the role of $\psi$ played by the coordinate $R$. Ignoring derivatives the null surface lies at the location $e^{-R}=a$. While derivatives are nonzero, they are of order $\frac{1}{d}$. It follows that the true null surface deviates from $e^{-R}=a$ only at order $\frac{1}{d}$. In other words we have not merely established our result; we have also determined the precise location of the event horizon at leading order in $\frac{1}{d}$ : it is simply the event horizon of the local black brane.

\section{A.4 Equivalence in data off the membrane}

In this short appendix we argue that the replacement

$$
O \rightarrow O+\delta O, \quad B \rightarrow \alpha B
$$


where $\delta O$ vanishes on the membrane, and $\alpha$ is any function that does not blow up or vanish on the membrane, generates equivalent metrics of the form (2.13).

In order to see this we note that in the large $d$ limit

$$
\frac{1}{\psi^{p+d}} \sim e^{-d(\psi-1)}
$$

As $\psi$ moves away from unity, in other words, this function dies off extremely rapidly. In other words the metric (2.13) only cares about the vector field $O$ in a neighbourhood of the membrane where $\psi-1=\mathcal{O}(1 / d)$. In this region the first Taylor expansion of $O-$ and the second Taylor expansion of $\psi$ - away from the membrane each contribute to the metric at order $\frac{1}{d}$. To leading order, in other words, the metric cares only about the location of the membrane, $O$ on the membrane, and $d \psi$ on the membrane.

We will now argue that $d \psi$ on the membrane is also completely determined by the shape of the membrane. $d \psi$ is proportional to the the normal vector field of the membrane surface. As a consequence it is fixed by the shape of the surface upto a position dependent normalization. Now the normalization of $\psi$ is unambiguously determined by the requirement $(S d \psi-d S) \cdot d S=0$. Indeed our definition of $\psi$ automatically obeys this equation. As the normalization of $\psi$ is fixed by a physical requirement, it follows that $d \psi$ is entirely determined by the shape of the membrane. ${ }^{28}$

We conclude that, to leading order, inequivalent ansatz are parametrized by the shape of the membrane and $O$ on the membrane.

\section{A.5 Details concerning Myers-Perry black holes}

The metric for a rotating black hole in arbitrary dimensions is well known [23, 24]. The black hole metric is given in Eddington-Finkelstein coordinates by in $2 N+2$ dimensions

$$
\begin{aligned}
d s^{2}= & 2\left(d v-\sum_{i=1}^{N} a_{i} \mu_{i}^{2} d \theta_{i}\right) d \rho+\sum_{i=1}^{N}\left(\rho^{2}+a_{i}^{2}\right)\left(d \mu_{i}^{2}+\mu_{i}^{2} d \theta_{i}^{2}\right)-d v^{2} \\
& +\left(\frac{m \rho}{\Pi F}\right)\left(d v-\sum_{i=1}^{N} a_{i} \mu_{i}^{2} d \theta_{i}\right)^{2}+\rho^{2} d \alpha^{2}
\end{aligned}
$$

while in $2 N+1$ dimensions the metric is given by

$$
\begin{aligned}
d s^{2}= & 2\left(d v-\sum_{i=1}^{N} a_{i} \mu_{i}^{2} d \theta_{i}\right) d \rho+\sum_{i=1}^{N}\left(\rho^{2}+a_{i}^{2}\right)\left(d \mu_{i}^{2}+\mu_{i}^{2} d \theta_{i}^{2}\right)-d v^{2} \\
& +\left(\frac{m \rho}{\Pi F}\right)\left(d v-\sum_{i=1}^{N} a_{i} \mu_{i}^{2} d \theta_{i}\right)^{2}
\end{aligned}
$$

In both (A.11) and (A.12), the black hole is characterized by a mass parameter $m$ and the $N$ independent angular velocities $a_{i}$ in the $N$ angular directions $\theta_{i}$. Apart from $\theta_{i}$, the

\footnotetext{
${ }^{28}$ The fact that the normalization of $d \psi$ is physical is closely related to the following observation. $d \psi$ is proportional to $d B$. Under the rescaling $B \rightarrow \alpha B, d B \rightarrow \alpha d B$ on the membrane. However $d \psi$ is unaffected by this scaling on the membrane.
} 
coordinates in both the metrics above consist of $\rho, v$ the $N$ 'direction cosines' $\mu_{i}$ yielding of total of $2 N+2$ coordinates in all. Of course this is one coordinate too many for the odd dimensional metric, in which case the coordinates $\mu_{i}$ are constrained to obey

$$
\sum_{i}^{N} \mu_{i}^{2}=1
$$

In the case of the even dimensional black hole, the additional 'coordinate' $\alpha$ in (A.11) is given in terms of direction cosines by

$$
\alpha^{2}+\sum_{i}^{N} \mu_{i}^{2}=1
$$

In both metrics the quantities $\Pi$ and $F$ are defined by

$$
\begin{aligned}
& \Pi=\prod_{i=1}^{N}\left(\rho^{2}+a_{i}^{2}\right), \\
& F=1-\sum_{i=1}^{N} \frac{a_{i}^{2} \mu_{i}^{2}}{\rho^{2}+a_{i}^{2}}
\end{aligned}
$$

The rotating black holes can be recast in 'flat space' type coordinates as follows. In the case of the even dimensional black hole we move to the new coordinates $x_{i}, y_{i}, z, t$ $(i=1 \ldots N)$ defined by

$$
\begin{aligned}
x_{i} & =\mu_{i}\left(\rho \cos \theta_{i}-a_{i} \sin \theta_{i}\right), \\
y_{i} & =\mu_{i}\left(\rho \sin \theta_{i}+a_{i} \cos \theta_{i}\right), \\
z & =\rho \alpha, \\
t & =v-\rho
\end{aligned}
$$

In the case of the odd dimensional black hole we use use the coordinates $x_{i}, y_{i}$ and $t$ defined in a similar manner by

$$
\begin{aligned}
x_{i} & =\mu_{i}\left(\rho \cos \theta_{i}-a_{i} \sin \theta_{i}\right), \\
y_{i} & =\mu_{i}\left(\rho \sin \theta_{i}+a_{i} \cos \theta_{i}\right), \\
t & =v-\rho
\end{aligned}
$$

In terms of the new coordinates the even dimensional black hole A.11 takes the form

$$
\begin{aligned}
d s^{2}= & -d t^{2}+\sum_{i=1}^{N}\left(d x_{i}^{2}+d y_{i}^{2}\right)+d z^{2} \\
& +\frac{m \rho}{\Pi F}\left(d t+\left(1-\sum_{i=1}^{N} \frac{\mu_{i}^{2} a_{i}^{2}}{\rho^{2}+a_{i}^{2}}\right) d \rho-\sum_{i=1}^{N} \frac{a_{i}}{\rho^{2}+a_{i}^{2}}\left(x_{i} d y_{i}-y_{i} d x_{i}\right)\right)^{2} .
\end{aligned}
$$


whereas the odd dimensional black hole A.12 turns into

$$
\begin{aligned}
d s^{2}= & -d t^{2}+\sum_{i=1}^{N}\left(d x_{i}^{2}+d y_{i}^{2}\right) \\
& +\frac{m \rho}{\Pi F}\left(d t+\left(1-\sum_{i=1}^{N} \frac{\mu_{i}^{2} a_{i}^{2}}{\rho^{2}+a_{i}^{2}}\right) d \rho-\sum_{i=1}^{N} \frac{a_{i}}{\rho^{2}+a_{i}^{2}}\left(x_{i} d y_{i}-y_{i} d x_{i}\right)\right)^{2} .
\end{aligned}
$$

The quantities $\mu_{i}$ and $\rho$ in (A.18) and (A.19) are now functions of our new coordinates. In the even dimensional case these qantities are determined by by the equations

$$
\begin{aligned}
& \mu_{i}^{2}=\frac{x_{i}^{2}+y_{i}^{2}}{\rho^{2}+a_{i}^{2}} \\
& \sum_{i=1}^{N} \frac{x_{i}^{2}+y_{i}^{2}}{\rho^{2}+a_{i}^{2}}+\frac{z^{2}}{\rho^{2}}=1
\end{aligned}
$$

while in the odd dimensional case the relevant relations are

$$
\begin{aligned}
& \mu_{i}^{2}=\frac{x_{i}^{2}+y_{i}^{2}}{\rho^{2}+a_{i}^{2}} \\
& \sum_{i}^{N} \frac{x_{i}^{2}+y_{i}^{2}}{\rho^{2}+a_{i}^{2}}=1
\end{aligned}
$$

In this paper we are interested in the special case of rotating black holes with non-zero rotations turned on in only $q$ two planes with $q \ll D$. Given such a black hole (as mentioned in the main text) we choose $p=2 q-1$. Let us now change notation so that $x_{i}$ and $y_{i}$ are coordinates of the two planes with non-zero rotations, i.e. $i=1 \ldots q$. We group the other $x_{i}, y_{i}$ coordinates (the coordinates of the remaining two planes in which the angular velocities all vanish) together as $z_{M}$ coordinates. In the even dimensional context (A.18) these $z_{M}$ coordinates appear on equal footing with the coordinate $z$ and we group that coordinate together with $z_{M}$. With these definitions the index $M$ runs over $d+1$ values and the black hole metric, in both odd and even dimensions, takes the form

$$
\begin{aligned}
d s^{2}= & -d t^{2}+\sum_{i=1}^{q}\left(d x_{i}^{2}+d y_{i}^{2}\right)+\sum_{M} d z_{M}^{2} \\
& +\frac{m \rho}{\Pi F}\left(d t+\left(1-\sum_{i=1}^{q} \frac{\mu_{i}^{2} a_{i}^{2}}{\rho^{2}+a_{i}^{2}}\right) d \rho-\sum_{i=1}^{q} \frac{a_{i}}{\rho^{2}+a_{i}^{2}}\left(x_{i} d y_{i}-y_{i} d x_{i}\right)\right)^{2} .
\end{aligned}
$$

where $\mu_{i}^{2}=\frac{x_{i}^{2}+y_{i}^{2}}{\rho^{2}+a_{i}^{2}}$ as above, and the equation that implicitly determines $\rho$ is

$$
\sum_{i=1}^{\left[\frac{p+1}{2}\right]} \frac{x_{i}^{2}+y_{i}^{2}}{\rho^{2}+a_{i}^{2}}+\frac{S^{2}}{\rho^{2}}=1
$$

where $S^{2}=\sum_{M=1}^{d+1} z_{M}^{2}$ as in the main text. 


\section{B Details of the perturbative procedure}

We move to a patch centred around $x_{0}^{\mu}$ and use the following patch coordinates

$$
\begin{aligned}
\frac{R}{d} & =(\psi-1), \quad \frac{V}{d}=e^{h\left(x_{0}\right)} O_{\mu}\left(x_{0}\right)\left(x^{\mu}-x_{0}^{\mu}\right), \quad \frac{Y}{d}=S-s_{0} \psi \\
\frac{y^{i}}{d} & =\mathcal{P}_{\mu}^{i}\left(x_{0}\right)\left(x^{\mu}-x_{0}^{\mu}\right)
\end{aligned}
$$

In these coordinates the rescaled zeroth order metric $G_{a b}$ and the one form $\chi$ take the following form.

$$
\begin{aligned}
d s^{2}= & 2 s_{0} e^{-h_{0}} d R d V-e^{-2 h_{0}} F(R) d V^{2} \\
& +\frac{d Y^{2}}{c^{2}}+\sum_{i=1}^{p} d y^{i} d y^{i}+\mathcal{O}\left(\frac{1}{d}\right) \\
\chi= & \frac{2 d Y}{s_{0}}+2 d R+\mathcal{O}\left(\frac{1}{d}\right), \quad e^{-\phi}=\frac{1}{s_{0}^{2}}+\mathcal{O}\left(\frac{1}{d}\right)
\end{aligned}
$$

where

$$
F(R)=\left(\left(1-c^{2}\right)-e^{2 h_{0}-R}\right)
$$

Ignoring corrections of order $\frac{1}{d}$, (B.2) is the black brane metric. ${ }^{29}$

In these coordinates the terms listed in the second line onwards of (3.8) takes the form

$$
\begin{aligned}
\left.d s^{2}\right|_{\mathcal{O}\left(\frac{1}{d}\right)}= & \frac{e^{-R}}{d}\left[K_{1}(R) d V^{2}+2 K_{2}(R) d V d Y+K_{3}(R) d Y^{2}\right. \\
& +2 K_{4}(R) d R d Y+K_{5}(R) d y^{i} d y^{i} \\
& \left.+2 Q_{1}^{i}(R) d V d y^{i}+2 Q_{1}^{i}(R) d Y d y^{i}+\mathcal{T}_{i j}(R) d y^{i} d y^{j}\right]
\end{aligned}
$$

As we have explained in the main text, at $\mathcal{O}\left(\frac{1}{d}\right)$ the scalar, vector and the tensor data are effectively constants (equal to their values at the point $x_{0}^{\mu}$ ) as derivatives of these expressions contribute to the metric only at $\mathcal{O}\left(\frac{1}{d}\right)^{2}$ and higher. It follows that

$$
\begin{aligned}
& K_{a}(R)=\sum_{A=0}^{7} K_{a}^{A}(R) S_{A}\left(x_{0}^{\mu}\right) \\
& Q_{a}^{i}(R)=\sum_{A=1}^{5} Q_{a}^{A}(R) V_{A}^{i}\left(x_{0}^{\mu}\right) \\
& \mathcal{T}_{i j}(R)=\sum_{A=1}^{2} \mathcal{T}_{A}(R) T_{A}^{i j}\left(x_{0}^{\mu}\right)
\end{aligned}
$$

${ }^{29}$ (B.2) can be cast into the standard form (2.10) by the rescaling $V=\left(\frac{e^{h_{0}}}{s_{0}}\right) \tilde{V}$ and shifting $R=$ $\tilde{R}+\left[2 h_{0}-\log \left(1-c^{2}\right)\right]$. The ' $a$ ' parameter of the standard form metric may be verified to be given by

$$
a=\frac{1-c^{2}}{s_{0}^{2}}
$$

In our computations below, however, we do not perform this scaling and shift; we work with the $V$ rather than the $\tilde{V}$ coordinate. 
Substituting (B.3) in Einstein equation and the scalar field equation as given in (2.2) we obtain ordinary differential equations for various metric functions. $\mathrm{SO}(p)$ symmetry ensures that the equations for the scalar functions $K_{a}$, vector functions $Q_{a}^{i}(R)$ and tensor functions $\mathcal{T}_{i j}(R)$ are mutually decoupled. Of course the equations for all functions of a given symmetry (e.g. scalars) all mix. Nonetheless it turns out that the equations are rather simple and explicitly solvable. ${ }^{30}$

Recall that our data included an antisymmetric tensor; as there is no antisymmetric tensor in the metric, it follows even without a calculation that there is no term in the correction metric proportional to this piece of data.

\section{B.1 Scalar sector}

In this sector the relevant equations are $E_{R R}, E_{R V}, E_{R Y}, E_{V V}, E_{V Y}, E_{Y Y}$ and $E_{t r} \equiv$ $\sum_{i} E_{i i}$. Here by $E_{\mu \nu}$ we denote the second equation in (2.2).

In order to solve these equations we found it useful to first study a linear combination of $E_{R V}$ and $E_{V V}$ which turns out to give a decoupled equation for $K_{2}(R)$.

$$
\begin{aligned}
E_{1} & \equiv s_{0} e^{h_{0}+R} E_{V V}+e^{R} F(R) E_{R V} \\
& =\sum_{A=0}^{7} S_{A}\left[\frac{c^{2} e^{R}}{2 s_{0}} F(R)^{2}\left[\frac{e^{-R} K_{2}^{A}(R)}{F(R)}\right]^{\prime}+\mathfrak{S}_{2}^{A}(R)\right]
\end{aligned}
$$

where' denotes the derivative w.r.t. $R$. The linear combination of equations presented in the first line (B.4) evaluates (by explicit computation) to the second line of that equation. The first term in (B.4) is homogeneous in the unknown $K_{2}^{A}(R)$ while the second term, $\mathfrak{S}_{2}^{A}(R)$, is the source term proportional to the scalar data $S^{A}$. The explicit expressions for these source terms are

$$
\begin{aligned}
& \mathfrak{S}_{2}^{0}(R)=-\frac{c^{2} e^{h_{0}}}{2 s_{0}} R \\
& \mathfrak{S}_{2}^{1}(R)=e^{h_{0}}\left(-\frac{c^{6}-6 c^{4}+8 c^{2}-1}{2 c^{2}\left(c^{2}-1\right)}+\frac{\left(c^{2}-2\right) R}{2\left(c^{2}-1\right)}-\frac{\left(c^{2}-2\right) e^{2 h_{0}-R}}{2\left(c^{2}-1\right)}\right) \\
& \mathfrak{S}_{2}^{2}(R)=\frac{e^{h_{0}}}{2}\left(2 c^{2}+\frac{1}{c^{2}}-6-R+\frac{\left(2 c^{2}-3\right) e^{2 h_{0}-R}}{c^{2}-1}\right) \\
& \mathfrak{S}_{2}^{3}(R)=\frac{e^{h_{0}}}{2}\left(1-c^{2}-e^{2 h_{0}-R}\right) \\
& \mathfrak{S}_{2}^{4}(R)=\frac{e^{h_{0}}}{2}, \quad \mathfrak{S}_{2}^{5}(R)=e^{h_{0}}\left(-2+c^{2}\right) \\
& \mathfrak{S}_{2}^{6}(R)=-2 \mathfrak{S}_{2}^{7}(R)=\left(1-c^{2}\right) e^{h_{0}}
\end{aligned}
$$

(B.4) is easily solved by integration and we obtain

$$
K_{2}^{A}(R)=\frac{2 s_{0} e^{R} F(R)}{c^{2}} \int_{R}^{\infty} d \rho\left(\frac{e^{-\rho} \mathfrak{S}_{2}^{A}(\rho)}{F(\rho)^{2}}\right)
$$

\footnotetext{
${ }^{30}$ As the equations for the unknowns are all linear it is possible in principle - and useful in practice to obtain solutions for pieces of one derivative background data turned on one at a time and then to add the results.
} 
The integration constant is fixed using the boundary condition that for large $R, K_{2}(R)$ should behave at most like a polynomial in $R$.

Now the function $F(R)$ has a zero at $R_{0}=2 h_{0}-\log \left(1-c^{2}\right)$. It follows that the integrand in (B.6) can be expanded around $\rho=R_{0}$ as

$$
\left(\frac{e^{-\rho} \mathfrak{S}_{2}^{A}(\rho)}{F(\rho)^{2}}\right)=\frac{B_{1}^{A}}{\left(\rho-R_{0}\right)^{2}}+\frac{B_{2}^{A}}{\rho-R_{0}}+\text { regular }
$$

Upon integrating, it follows that

$$
K_{2}(R) \propto\left(R-R_{0}\right) \sum_{A}\left(\frac{B_{1}^{A} S_{A}}{R-R_{0}}+B_{2}^{A} S_{A} \ln \left(R-R_{0}\right)+\text { regular }\right)
$$

While the terms proportional to $B_{1}^{A}$ above are perfectly analytic, the terms proportional to $B_{2}^{A}$ are potentially singular (non analytic). It follows that $K_{2}(R)$ solutions is regular everywhere outside its event horizon (as we demand) if and only if

$$
\sum_{A} B_{2}^{A} S_{A}=0
$$

This equation may be rewritten as

$$
\text { Equation of motion } \propto \sum_{A=0}^{7} S^{A}\left(\left.\partial_{R} \mathfrak{S}_{2}^{A}(R)\right|_{R=R_{0}}\right)=0
$$

upon explicitly evaluating the derivatives in (B.7) we find the scalar equation in (1.7). After imposing the equation of motion $K_{2}(R)$ is perfectly regular.

Next we solve $E_{R Y}=0$. If we substitute the solution for $K_{2}(R)$ and the equation of motion, $E_{R Y}$ becomes very simple:

$$
E_{R Y} \equiv \frac{c^{2}}{2 s_{0}} \partial_{R}\left(e^{-R} K_{3}(R)\right)=0
$$

The only normalizable solution to this equation

$$
K_{3}(R)=0
$$

$E_{t r}$ gives another decoupled source free equation for $K_{5}(R)$.

$$
E_{t r} \equiv \frac{e^{-R}}{2 s 0^{2}} \partial_{R}\left(e^{R} F(R) \partial_{R}\left(e^{-R} K_{5}(R)\right)\right)=0
$$

If we impose regularity at $R=R_{0}$ and normalizability at $R=\infty$ the only allowed solution is

$$
K_{5}(R)=0
$$

After we substitute the solutions for $K_{2}(R), K_{3}(R)$ and $K_{5}(R)$, the equation $E_{R R}$ becomes the simple source free equation for $K_{4}(R)$.

$$
E_{R Y} \equiv \frac{c^{2}}{2 s_{0}} \partial_{R}\left(e^{-R} K_{4}(R)\right)=0
$$


From here it follows

$$
K_{4}(R)=0
$$

Finally we solve for $K_{1}(R)$. We use the scalar field equation (i.e., the first equation in (2.2)) for this purpose. After substituting in the solution for all the other $K_{i}(R)$ as well as the equation of motion, this scalar equation takes the form

$$
E_{\phi} \equiv \sum_{A=0}^{7} S_{A}\left(\partial_{R} K_{1}^{A}(R)+\mathfrak{S}_{1}^{A}(R)\right)=0
$$

where

$$
\begin{aligned}
& \mathfrak{S}_{1}^{0}(R)=\frac{-c^{6}+11 c^{4}-23 c^{2}+11+\left(c^{2}-1\right)^{2}\left(3 c^{2}-8\right) R}{\left(c^{2}-2\right)^{2}\left(c^{2}-1\right)} \\
& \mathfrak{S}_{1}^{1}(R)=0 \\
& \mathfrak{S}_{1}^{2}(R)=\frac{2 s_{0}\left(1-2 c^{4}+c^{2}(R+3)\right)}{c^{2}\left(c^{4}-3 c^{2}+2\right)} \\
& \mathfrak{S}_{1}^{3}(R)=\frac{s_{0}\left(4 c^{4}-c^{2}(R+7)-1\right)}{c^{2}\left(c^{2}-2\right)^{2}} \\
& \mathfrak{S}_{1}^{4}(R)=\frac{s_{0}}{1-c^{2}}, \quad \mathfrak{S}_{1}^{5}(R)=\frac{2 s_{0}}{c^{2}-1} \\
& \mathfrak{S}_{1}^{6}(R)=\mathfrak{S}_{1}^{7}(R)=0
\end{aligned}
$$

(We have used the equation of motion to solve for $S_{1}$ in terms of the other scalar data. That is why $\left.\mathfrak{S}_{1}^{1}(R)=0\right)$.

Equation (B.11) is trivially solved by integration and we find

$$
K_{1}^{A}(R)=-\int_{0}^{R} d \rho \mathfrak{S}_{1}^{A}(\rho) .
$$

We have chosen the integration constant above to satisfy the arbitrary condition $K_{1}^{A}(R=$ $0)=0$. A different choice of integration constant could be absorbed by a shift of $\psi$ by a function of order $\frac{1}{d}$ and so is physically irrelevant.

\section{B.2 Vector sector}

Here the relevant equations are $E_{R y^{i}}, E_{V y^{i}}$ and $E_{Y y^{i}}$. We first study the linear combination of $E_{R y^{i}}$ and $E_{V y^{i}}$ which yields a decoupled equation for $Q_{2}^{A}(R)$.

$$
\begin{aligned}
E_{i} & \equiv 2\left(e^{h_{0}} s_{0}^{2} E_{V y^{i}}+s_{0} F(R) E_{R y^{i}}\right) \\
& =\sum_{A=1}^{5} S_{A}\left(c^{2} F(R) \partial_{R}\left(e^{-R} Q_{2}^{A}(R)\right)+\mathfrak{V}_{2}^{A}(R)\right)=0
\end{aligned}
$$

where

$$
\begin{aligned}
& \mathfrak{V}_{2}^{1}(R)=-s_{0}\left(2-c^{2}\right) e^{2 h_{0}-R} \\
& \mathfrak{V}_{2}^{2}(R)=s_{0}\left(1-c^{2}\right) e^{2 h_{0}-R} \\
& \mathfrak{V}_{2}^{3}(R)=0, \quad \mathfrak{V}_{2}^{4}(R)=0, \quad \mathfrak{V}_{2}^{5}(R)=0
\end{aligned}
$$


Solving this equation with the boundary condition of 'normalizability' at infinity we find

$$
Q_{2}^{A}(R)=\frac{e^{R}}{c^{2}} \int_{R}^{\infty} d \rho\left(\frac{\mathfrak{V}_{2}^{A}(\rho)}{F(\rho)}\right)
$$

Clearly $Q_{2}^{A}(R)$ above has a logarithmic singularity at $R=R_{0}$; the coefficient of this singularity is proportional to the vector equation of motion

$$
\text { Equation of Motion } \equiv \sum_{A=1}^{5} V_{A}^{\mu} \mathfrak{V}_{2}^{A}\left(R_{0}\right)=0
$$

Upon substitution, (B.16) reduces to the second of (1.7). Note, however, that all the nonzero source functions $\mathfrak{V}_{2}^{A}(R)$ are proportional to each other as functions of $R$ (they are all proportional to $e^{-R}$ ). It follows that (B.16) implies that

$$
\sum_{A=1}^{5} V_{A}^{\mu} \mathfrak{V}_{2}^{A}(R)=0
$$

at all $R$ and so it follows from (B.15) that

$$
Q_{2}(R)=0
$$

Upon substituting the solution for $Q_{2}(R)$ and also the equation of motion, the equation $E_{R y^{i}}$ allows us to solve for $Q_{1}(R)$. It turns out that only $Q_{1}^{1}(R)$ has a nonzero source and we find

$$
\frac{e^{h_{0}-R}}{4 s_{0}} \partial_{R}\left(e^{R} \partial_{R}\left(e^{-R} Q_{1}^{1}(R)\right)\right)+\frac{e^{2 h_{0}-R}}{2\left(1-c^{2}\right)}=0
$$

(B.17) is easily solved by two integrations. The solution is automatically regular everywhere. There are two integration constants. One of these is fixed by the requirement of 'normalizability' at $R=\infty$. The other integration constant can be absorbed into a shift in velocity field $u^{\mu}$ by a term of order $\frac{1}{d}$. We have arbitrarily fixed it by demanding that $Q_{1}(0)=0$.

\section{B.3 Tensor sector}

The equations are simplest in the symmetric tensor sector. The unique tensor equation, $E_{y^{i} y^{j}}$, evaluates to

$$
E^{y^{i} y^{j}} \equiv-\sum_{A=1}^{2}\left[T_{A}\right]^{y^{i} y^{j}} e^{-R} \partial_{R}\left(e^{R} F(R) \partial_{R}\left(e^{-R} \mathcal{T}_{A}(R)\right)\right)=0
$$

Quite remarkably no tensor source appears on the r.h.s. of (B.18). The most general solution of this equation is easily determined, and turns out to be either non-normalizable or singular at $R=R_{0}$. It follows that

$$
\mathcal{T}_{A}(R)=0
$$

and there are no corrections to the metric in the tensor sector. 
Open Access. This article is distributed under the terms of the Creative Commons Attribution License (CC-BY 4.0), which permits any use, distribution and reproduction in any medium, provided the original author(s) and source are credited.

\section{References}

[1] G. 't Hooft, A Planar Diagram Theory for Strong Interactions, Nucl. Phys. B 72 (1974) 461 [INSPIRE].

[2] E. Witten, Quarks, atoms, and the 1/N expansion, Phys. Today 33 (1980) 38.

[3] R. Emparan, R. Suzuki and K. Tanabe, The large D limit of General Relativity, JHEP 06 (2013) 009 [arXiv: 1302.6382] [INSPIRE].

[4] R. Emparan, D. Grumiller and K. Tanabe, Large-D gravity and low-D strings, Phys. Rev. Lett. 110 (2013) 251102 [arXiv:1303.1995] [INSPIRE].

[5] R. Emparan and K. Tanabe, Holographic superconductivity in the large D expansion, JHEP 01 (2014) 145 [arXiv:1312.1108] [INSPIRE].

[6] R. Emparan and K. Tanabe, Universal quasinormal modes of large D black holes, Phys. Rev. D 89 (2014) 064028 [arXiv:1401.1957] [InSPIRE].

[7] R. Emparan, R. Suzuki and K. Tanabe, Instability of rotating black holes: large D analysis, JHEP 06 (2014) 106 [arXiv:1402.6215] [INSPIRE].

[8] R. Emparan, R. Suzuki and K. Tanabe, Decoupling and non-decoupling dynamics of large D black holes, JHEP 07 (2014) 113 [arXiv: 1406.1258] [INSPIRE].

[9] R. Emparan, R. Suzuki and K. Tanabe, Quasinormal modes of (Anti-)de Sitter black holes in the 1/D expansion, JHEP 04 (2015) 085 [arXiv: 1502.02820] [INSPIRE].

[10] R. Emparan, Black Holes in 1/D expansion, talk given at Eurostrings 2015, Cambridge, U.K., 23-27 March 2015.

[11] S. Bhattacharyya, V.E. Hubeny, S. Minwalla and M. Rangamani, Nonlinear Fluid Dynamics from Gravity, JHEP 02 (2008) 045 [arXiv:0712.2456] [INSPIRE].

[12] M. Rangamani, Gravity and Hydrodynamics: Lectures on the fluid-gravity correspondence, Class. Quant. Grav. 26 (2009) 224003 [arXiv:0905.4352] [INSPIRE].

[13] V.E. Hubeny, S. Minwalla and M. Rangamani, The fluid/gravity correspondence, arXiv:1107.5780 [INSPIRE].

[14] J. Armas, (Non)-Dissipative Hydrodynamics on Embedded Surfaces, JHEP 09 (2014) 047 [arXiv: 1312.0597] [INSPIRE].

[15] R. Emparan, T. Harmark, V. Niarchos and N.A. Obers, Blackfolds in Supergravity and String Theory, JHEP 08 (2011) 154 [arXiv: 1106.4428] [INSPIRE].

[16] J. Camps and R. Emparan, Derivation of the blackfold effective theory, JHEP 03 (2012) 038 [Erratum ibid. 1206 (2012) 155] [arXiv:1201.3506] [INSPIRE].

[17] J. Armas, How Fluids Bend: the Elastic Expansion for Higher-Dimensional Black Holes, JHEP 09 (2013) 073 [arXiv:1304.7773] [INSPIRE].

[18] R. Emparan, T. Shiromizu, R. Suzuki, K. Tanabe and T. Tanaka, Effective theory of Black Holes in the 1/D expansion, JHEP 06 (2015) 159 [arXiv: 1504.06489] [INSPIRE]. 
[19] T. Damour, Black Hole Eddy Currents, Phys. Rev. D 18 (1978) 3598 [inSPIRE].

[20] R.H. Price and K.S. Thorne, The membrane paradigm for black holes, Sci. Am. 258 (1988) 69.

[21] I. Bredberg, C. Keeler, V. Lysov and A. Strominger, Wilsonian Approach to Fluid/Gravity Duality, JHEP 03 (2011) 141 [arXiv: 1006.1902] [INSPIRE].

[22] S. Bhattacharyya et al., Local Fluid Dynamical Entropy from Gravity, JHEP 06 (2008) 055 [arXiv: 0803.2526] [INSPIRE].

[23] R.C. Myers and M.J. Perry, Black Holes in Higher Dimensional Space-Times, Annals Phys. 172 (1986) 304 [INSPIRE].

[24] R.C. Myers, Myers-Perry black holes, arXiv:1111.1903 [INSPIRE]. 